\title{
1 Revealing the impact of recurrent and rare structural 2 variants in multiple myeloma
}

Even H Rustad ${ }^{1}$, Venkata D Yellapantula ${ }^{1}$, Dominik Glodzik ${ }^{2}$, Kylee H Maclachlan ${ }^{1}$, Benjamin Diamond ${ }^{1}$, Eileen M Boyle ${ }^{3}$, Cody Ashby ${ }^{4}$, Patrick Blaney ${ }^{3}$, Gunes Gundem², Malin Hultcrantz ${ }^{1}$, Daniel Leongamornlert ${ }^{5}$, Nicos Angelopoulos ${ }^{6}$, Daniel Auclair ${ }^{7}$, Yanming Zhang ${ }^{8}$, Ahmet Dogan ${ }^{9}$, Niccolò Bolli ${ }^{10-11}$, Elli Papaemmanuil ${ }^{2}$, Kenneth C. Anderson $^{12}$, Philippe Moreau ${ }^{13}$, Herve Avet-Loiseau ${ }^{14}$, Nikhil Munshi ${ }^{12,15}$, Jonathan Keats $^{16}$, Peter J Campbell ${ }^{5}$, Gareth J Morgan ${ }^{3}$, Ola Landgren ${ }^{1}$ and Francesco Maura ${ }^{1}$

1 Myeloma Service, Department of Medicine, Memorial Sloan Kettering Cancer Center, New York, NY, USA;

2 Epidemiology \& Biostatistics, Department of Medicine, Memorial Sloan Kettering Cancer Center, New York, NY, USA;

3 NYU Perlmutter Cancer Center, New York, NY, USA; 4 Myeloma Center, University of Arkansas for Medical Sciences, Little Rock, AR, USA; 5 The Cancer, Ageing and Somatic Mutation Programme, Wellcome Sanger Institute, Hinxton, Cambridgeshire, United Kingdom;

6 School of Computer Science and Electronic Engineering, University of Essex, Colchester, United Kingdom;

7 Multiple Myeloma Research Foundation (MMRF), Norwalk, US-CT;

8 Cytogenetics Laboratory, Department of Pathology, Memorial Sloan Kettering Cancer Center, New York, NY, USA;

9 Hematopathology Service, Department of Pathology, Memorial Sloan Kettering Cancer Center, New York, NY, USA;

10 Department of Medical Oncology and Hemato-Oncology, University of Milan, Milan, Italy;

11 Department of Medical Oncology and Hematology, Fondazione IRCCS Istituto Nazionale dei Tumori, Milan, Italy;

12 Jerome Lipper Multiple Myeloma Center, Dana-Farber Cancer Institute, Harvard Medical School, Boston, MA; 13 CRCINA, INSERM, CNRS, Université d'Angers, Université de Nantes, Nantes, France; 14 IUC-Oncopole, and CRCT INSERM U1037, 31100, Toulouse, France; 15 Veterans Administration Boston Healthcare System, West Roxbury, MA; 16. Translational Genomics Research Institute (TGen), Phoenix, AZ, USA;

Running Title: Impact of structural variants in myeloma

Key words: Multiple Myeloma, structural variants, hotspots, chromothripsis, templated insertion, chromoplexy. 
bioRxiv preprint doi: https://doi.org/10.1101/2019.12.18.881086; this version posted December 19, 2019. The copyright holder for this preprint (which was not certified by peer review) is the author/funder. All rights reserved. No reuse allowed without permission.

46 Corresponding Author:

47 Francesco Maura, MD,

48 Myeloma Service, Department of Medicine

49 Memorial Sloan Kettering Cancer Center, 1275 York Ave, New York, NY 10065

$50 \quad \mathrm{~T}:+1646.608 .3934 \mid \mathrm{F}:+1646.277 .7116$

51 mauraf@mskcc.org

52 


\section{Summary}

54 The landscape of structural variants $(\mathrm{SVs})$ in multiple myeloma remains poorly

55 understood. Here, we performed comprehensive classification and analysis of SVs in

56 multiple myeloma, interrogating a large cohort of 762 patients with whole genome and

57 RNA sequencing. We identified $100 \mathrm{SV}$ hotspots involving 31 new candidate driver

58 genes, including drug targets BCMA (TNFRSF17) and SLAMF7. Complex SVs,

59 including chromothripsis and templated insertions, were present in $61 \%$ of patients and

60 frequently resulted in the simultaneous acquisition of multiple drivers. After accounting

61 for all recurrent events, $63 \%$ of SVs remained unexplained. Intriguingly, these rare SVs

62 were associated with up to 7-fold enrichment for outlier gene expression, indicating that

63 many rare driver SVs remain unrecognized and are likely important in the biology of

64 individual tumors.

65 
66

67

68

69

\section{Introduction}

Structural variations (SVs) are increasingly recognized as key drivers of cancer development ${ }^{1-3}$. Functional implications of SVs are diverse, including large-scale gain and loss of chromosomal material, gene regulatory effects such as super-enhancer hijacking, and gene fusions ${ }^{4}$. The basic unit of SVs are pairs of breakpoints, classified as either deletion, tandem duplication, translocation or inversion ${ }^{5}$. Recently, whole genome sequencing (WGS) studies have revealed complex patterns where multiple SVs are acquired together in a single event, often involving multiple chromosomes, with a potentially important role in cancer initiation and progression ${ }^{2,6-12}$. In multiple myeloma, chromosomal translocations are considered to be initiating events in $\sim 40 \%$ of patients, where the immunoglobulin heavy chain $(I G H)$ locus on chromosome 14 is juxtaposed with a class-defining oncogene ${ }^{13}$. Translocations involving MYC have been identified in 15-42\% of newly diagnosed multiple myeloma patients and are associated with progression to multiple myeloma from its precursor stage, smoldering multiple myeloma ${ }^{14-17}$. Recurrent translocations involving the immunoglobulin lambda $(I G L)$ locus and other genes have recently been reported, expanding the catalogue of potential drivers and confirming the critical role of SVs in multiple myeloma pathogenesis ${ }^{14}$.

The genomic landscape of multiple myeloma is characterized by multiple aneuploidies, which fall into two main categories ${ }^{13,18,19}$. First, whole chromosome gains of more than two odd-number chromosomes is referred to as hyperdiploidy (HRD) and present in $\sim 60 \%$ of patients ${ }^{20}$. HRD is considered to be the initiating event in most of the patients without a canonical $I G H$-translocation, with some patients acquiring additional 
89 whole chromosome gains later in tumor evolution ${ }^{13,20}$. The second category is made up of

90 all other recurrent copy number alterations (CNAs), one or more of which are present in

91 virtually all patients ${ }^{19,20}$. Among these, gain of chromosome 1q21 often occurs early in

92 tumor evolution, while loss of tumor suppressor genes tends occur later ${ }^{20-23}$. The

93 structural basis of these established drivers remains poorly understood, and there is a lack

94 of data about the role of SVs beyond the most recurrent aberrations.

95 We recently reported the first comprehensive SV characterization using WGS of

96 sequential samples from 30 multiple myeloma patients ${ }^{20}$. Despite the small sample set,

97 SVs emerged as key drivers during all evolutionary phases of disease, and we identified a

98 high prevalence of three main classes of complex SVs: chromothripsis, templated

99 insertions and chromoplexy ${ }^{20}$. In chromothripsis, chromosomal shattering and random

100 rejoining results in a pattern of tens to hundreds of breakpoints with oscillating copy

101 number across one or more chromosomes ${ }^{24}$. Templated insertions are characterized by

102 focal gains bounded by translocations, resulting in concatenation of amplified segments

103 from two or more chromosomes into a continuous stretch of DNA, which is inserted back

104 into any of the involved chromosomes ${ }^{2,20}$. Chromoplexy similarly connects segments

105 from multiple chromosomes, but the local footprint is characterized by copy number

$106 \operatorname{loss}^{25}$. Importantly, complex SVs represent large-scale genomic alterations acquired by

107 the cancer cell at a single point in time, potentially driving subsequent tumor evolution.

108 Here, we present the first comprehensive study of SVs in a large series of 762

109 multiple myeloma patients. We show that recurrent and rare SVs are critical in shaping

110 the genomic landscape of multiple myeloma, including complex events simultaneously

111 causing multiple drivers. 


\section{Results}

\section{Genome-wide landscape of structural variation in multiple myeloma}

115 To define the landscape of simple and complex SVs in multiple myeloma, we

116 investigated 762 newly diagnosed patients from the CoMMpass study (NCT01454297)

117 who underwent low coverage long-insert WGS (median 4-8X) (Table S1). RNA

118 sequencing was also available from 592 patients. As a validation cohort, we compiled

119 previously published WGS data from 52 patients $^{20,26,27}$. For each patient sample, we

120 integrated the genome-wide somatic copy number profile with SV data and assigned each

121 pair of SV breakpoints as either simple or part of a complex event according to the three

122 main classes previously identified in multiple myeloma (Methods) ${ }^{20}$. Templated

123 insertions involving more than two chromosomes were considered complex. Events

124 involving more than three breakpoint pairs which did not fulfill the criteria for a specific

125 class of complex event were classified as unspecified "complex"20.

126 Overall, we identified a median of 15 SVs per patient in the CoMMpass cohort

127 (interquartile range, IQR 7-28) (Figure 1A). Deletion was the most common SV type (33

$128 \%$ ), followed by inversion and translocation (24\% each), and tandem duplication (18\%)

129 (Figure S1A). Fifty-one percent of SVs (8842/17185) were defined as part of 931

130 complex events (Figure 1A). Chromothripsis, chromoplexy and templated insertions

131 involving $>2$ chromosomes were observed in $21 \%, 11 \%$ and $21 \%$ of patients,

132 respectively; $32 \%$ of patients had an unspecified complex event. One or more complex

133 events were identified in $61 \%$ of patients (median 1 ; range $0-14$ ); $16 \%$ had two complex

134 SVs; $14 \%$ had three or more. The distribution across simple and complex classes varied 
135 for each SV type: deletions were most commonly simple events (71\%), followed by 18

$136 \%$ attributed to chromothripsis; while translocations were simple events in $28 \%$ (e.g.

137 reciprocal or unbalanced) and part of templated insertions or chromothripsis in $31 \%$ each

138 (Figure S1A). In the validation cohort we identified a slightly higher overall SV burden,

139 with a median of $26 \mathrm{SVs}$ per patient (IQR 10-36), as expected from the higher sequencing

140 coverage. Reassuringly, the distribution across simple and complex SV classes was

141 similar across the datasets (Figure S1A), suggesting that low-coverage long-insert WGS

142 provides a representative view of the SV landscape.

143 SV breakpoints tend to be enriched within specific genomic contexts, depending

144 on the underlying mechanism. Our findings from multiple myeloma corresponded with

145 previous reports from pan-cancer studies ${ }^{2,11}$. A higher density of SV breakpoints was

146 generally associated with early replication, chromatin accessibility (DNAse seq), highly

147 expressed genes and active enhancer regions as defined by histone H3K27 acetylation

148 (H3K27ac) (Figure S1B; Methods). These associations were particularly strong for the

149 breakpoints of tandem duplications and templated insertions, which also showed a strong

150 correlation with each other (Spearman's rho $=0.33, \mathrm{p}<0.001$ ). Turning to the SV burden

151 in individual patients, the number of templated insertion breakpoints was inversely

152 correlated with all other SV classes, except tandem duplications, which showed a striking

153 positive correlation (Spearman's rho $=0.46, \mathrm{p}<0.001)$ (Figure S1C). Taken together,

154 templated insertions and tandem duplications tended to occur in the same genomic

155 contexts, and to be enriched in the same tumors.

156 In patients with newly diagnosed multiple myeloma, complex SV classes showed

157 distinct patterns of co-occurrence, mutual exclusivity and association with recurrent 
158 molecular alterations (Figures 1A and S2). High APOBEC mutational burden, a feature

159 associated with aggressive disease and poor outcomes ${ }^{28,29}$, was enriched in patients with

160 chromothripsis $(\mathrm{OR}=2.8,95 \%$ CI $1.8-4.3 ; \mathrm{p}<0.001)$ and unspecified complex events

$161(\mathrm{OR}=2.6,95 \%$ CI $1.7-4.0 ; \mathrm{p}<0.001)$, but was mutually exclusive with templated

162 insertions $(\mathrm{OR}=0.49,95 \%$ CI $0.29-0.80 ; \mathrm{p}=0.003)$. Chromothripsis was the only SV

163 class with clear prognostic implications after adjustment for molecular and clinical

164 features, resulting in adverse progression free survival (PFS, adjusted HR $=1.57 ; 95 \% \mathrm{CI}$

165 1.13-2.22; $\mathrm{p}=0.008)$ and overall survival $(\mathrm{OS}$, adjusted HR $=2.4 ; 95 \%$ CI 1.5-3.83; $\mathrm{p}<$

166 0.001) (Figure 1B-D; Methods) ${ }^{30}$.

167 Bi-allelic inactivation of TP53 was identified in 23 patients in our cohort, defining

168 a patient population with very poor prognosis ${ }^{21}$. Interestingly, these patients showed

169 strong enrichment for chromothripsis (15 out of 24 ; OR 7.8, p $<0.001)$ and high

170 APOBEC mutational burden (OR 6.6, $\mathrm{p}<0.001)$ (Figure $1 \mathbf{A}$ and $\mathbf{S 2}$ ). In 3 of 15 cases,

171 the TP53 deletion was caused by a chromothripsis event; and the association remained

172 highly significant after excluding these cases (OR 6.2, $\mathrm{p}<0.001)$. This relationship did

173 not reach statistical significance for monoallelic TP53 events. Consistent with our

174 findings, an association between chromothripsis and TP53 inactivation has been reported

175 in several other cancers ${ }^{31-33}$.

\section{Structural basis of recurrent translocations and copy number variants}

178 To define the structural basis of canonical translocations in multiple myeloma, we

179 identified all translocation-type events (single and complex) with one or more

180 breakpoints involving the immunoglobulin loci (i.e. $I G H, I G K$ and $I G L$ ) or canonical 
181 IGH-partners (e.g. CCND1, MMSET and MYC) ${ }^{13}$. Templated insertions emerged as the

182 cause of $C C N D 1$ and $M Y C$ translocations in $34 \%$ and $73 \%$ of cases, respectively

183 (Figure 2A). This is particularly important given that templated insertions connect and

184 amplify distant genomic segments, often involving several oncogenes and regulatory

185 regions (e.g. super-enhancers). Indeed, templated insertions of CCND1 and MYC were

186 associated with focal amplification in $63 \%$ and $90 \%$ of cases, respectively; and involved

187 more than two chromosomes in $24 \%$ and $41 \%$ of cases. Although rare, we also found

188 examples of chromothripsis and chromoplexy underlying canonical $I G H$ translocations,

189 resulting in overexpression of the partner gene consistent with a driver event (Figure

190 2B). Twenty-four patients (3.2\%) had a translocation involving an immunoglobulin locus

$191(I G H=11, I G L=12$ and $I G K=1)$ and a non-canonical oncogene partner, most of which

192 are key regulators of B-cell development (e.g. PAX5 and CD40) (Figure 2B) ${ }^{34,35}$. One of

193 the patients with non-canonical $I G H$ translocation also had an IGH-MMSET

194 translocation; while the remaining 10 patients either had $\operatorname{HRD}(n=8)$ or no known

195 initiating event $(n=2)$, suggesting the disease may have been initiated by a non-canonical

$196 I G H$ translocation. Taken together, we show that different mechanisms of SV converge to

197 aberrantly activate key driver genes in multiple myeloma. Importantly, SVs with strong

198 biological implications and a potential role in tumor initiation are not necessarily

199 recurrent, even in a series as large as the one analyzed here.

200 Next, we addressed the structural basis of known recurrent CNAs (Table S2).

201 Aneuploidies involving a whole chromosome arm were most common (60 \% of 2926

202 events); $24 \%$ could be attributed to a specific SV and $14 \%$ were intrachromosomal

203 events without a clear causal SV, classified as "unknown" (Figure 2C). The proportion 
204 of CNAs explained by a SV was similar in the validation dataset (29\% of 450 events;

205 Fisher's test $p=0.41$; Table S3). There was considerable variation in the proportion and

206 class of SVs causing gains and losses between different loci, indicating the presence of

207 distinct underlying mechanisms being active at these sites (Figure 2C). Loss of

208 CDKN2C, FAM46C, 6q21, 6q25.3, MAX and TP53 could be attributed to SVs in $\sim 50 \%$

209 of cases. SVs accounted for $78 \%$ of BIRC2/BIRC3 losses, and $62 \%$ of $M Y C$ gains, of

210 which $34 \%$ were caused by templated insertions. Interestingly, unbalanced translocations

211 were responsible for $5.5 \%$ of all recurrent deletions $(n=132)$, up to $8 \%$ for $17 \mathrm{p}$ and 16

$212 \%$ for $6 q$. Finally, $44.5 \%$ of all chromothripsis events resulted in the acquisition of at

213 least one recurrent driver CNA $(n=94)$; the corresponding numbers for chromoplexy and

214 templated insertions involving $>2$ chromosomes were $41.6 \%(n=40)$ and $19.9 \%(n=$

215 42), respectively.

216 In $12.5 \%$ of patients $(n=95)$, two or more seemingly independent recurrent CNAs

217 or canonical translocations were caused by the same simple or complex SV (Figure 2D).

218 The most common event classes were chromothripsis $(n=33)$, chromoplexy $(n=22)$,

219 unbalanced translocation $(\mathrm{n}=21)$ and templated insertion $(\mathrm{n}=21)$ (Figure 3A-C).

220 Intriguingly, translocation of $M Y C$ occurred as part of the initiating $I G H$-translocation in

221 five patients, together with $C C N D 1(\mathrm{n}=3)$ or $C C N D 2(\mathrm{n}=2)$. Another interesting

222 phenomenon was observed on chromosome 6, where gain of one arm (p) and loss of the

223 other (q) resulted from a single inversion event in seven patients (Figure 3D). 
Twenty recurrently translocated regions have been previously reported in multiple

227 myeloma, defined by a translocation prevalence of $>2 \%$ within $1 \mathrm{Mb}$ bins across the

228 genome ${ }^{14}$. Recurrent regions included the immunoglobulin loci, canonical partner

229 oncogenes, as well recurrent $M Y C$ partners, such as BMP6/TXNDC5, FOXO3 and

$230 F A M 46 C^{14,17,36}$. We were motivated to expand the known catalogue of genomic loci

231 where SVs play a driver role in multiple myeloma and are therefore positively selected

232 (i.e. SV hotspots), considering all classes of single and complex SVs. To accomplish this,

233 we applied the Piecewise Constant Fitting (PCF) algorithm, comparing the local SV

234 breakpoint density to an empirical background model (Methods; Figure S3A-E; Data

235 S1 $)^{8,37}$. Overall, we identified $100 \mathrm{SV}$ hotspots after excluding the immunoglobulin loci

236 (i.e., $I G H, I G L$ and $I G K), 85$ of which have not been previously reported (Figure 4A;

237 Table S4). Each patient had a median of three hotspots involved by a SV (IQR 1-5); and

238 the number of hotspots involved was strongly associated with the overall SV burden

239 (Spearman's Rho $=0.51 ; \mathrm{p}<0.001 ;$ Figure S3F). Two of the previously reported regions

240 of recurrent translocation were not recapitulated by our hotspot analysis: a locus of

241 unknown significance on 19p13.3, and the known oncogene $M A F B$ on 20q12. This may

242 be explained by the behavior of the PCF algorithm, which favors the identification of loci

243 where breakpoints are tightly clustered compared with neighboring regions as well as the

244 expected background. Translocations involving $M A F B$ were identified in $1.6 \%$ of

245 patients in our series $(\mathrm{n}=12)$, but the breakpoints were widely spread out across $600 \mathrm{~Kb}$

246 in the intergenic region upstream of $M A F B^{38}$. Similarly, a $2 \mathrm{Mb}$ region on $19 \mathrm{p} 13.3$ was

247 involved in 44 patients (5.7\%), predominantly by templated insertions and tandem

248 duplications, but the breakpoints did not form a distinct cluster. 
Given that SVs and CNAs reflect the same genomic events, we hypothesized that

250 functionally important SV hotspots would be associated with a cluster of CNAs. We

251 therefore performed independent discovery of driver CNAs using GISTIC (genomic

252 identification of significant targets in cancer) $)^{39}$. This algorithm identifies peaks of copy

253 number gain or loss which are likely to contain driver genes and/or regulatory elements

254 based on the frequency and amplitude of observed CNAs (Figure 4A, Table S5-6). In

255 addition, we generated cumulative copy number profiles for the patients involved by SV

256 at each hotspot. Finally, we evaluated the impact of SV hotspots on the expression of

257 nearby genes. By integrating SV, CNA, and expression data, we went on to determine the

258 most likely consequence of each hotspot in terms of gain-of-function, loss-of-function,

259 and potential involvement of driver genes and regulatory elements (Figures 4A-D and

260 S4).

261 Gain-of-function hotspots were defined by copy number gains and/or

262 overexpression of a putative driver gene (Table S4 and Figures 4A-C, 5A and S4).

263 Hotspots defined by these criteria showed overwhelming dominance of tandem

264 duplication, translocation and templated insertion SV breakpoints (median $73 \%$ of

265 breakpoints [IQR 63-87\%] in gain-of-function hotspots vs $21 \%$ [11-28\%] in other

266 hotspots; Wilcoxon rank sum test $\mathrm{p}<0.001$; Figure 5B). By contrast, chromothripsis,

267 chromoplexy and unspecified complex events had a similar contribution to gain- and

268 loss-of-function hotspots (Wilcoxon rank sum test, Bonferroni-Holm adjusted p-values >

269 0.05). Strikingly, gain-of-function hotspots showed 5.5-fold enrichment of super-

270 enhancers as compared with the remaining mappable genome (1.6 vs 0.3 super-enhancers

271 per $\mathrm{Mb}$, Poisson test $\mathrm{p}<0.001)(\text { Methods })^{40}$. Enhancer peaks $(\mathrm{H} 3 \mathrm{~K} 27 \mathrm{ac})$ clustered 
272 around the SV breakpoints were observed in 46 out of 62 gain-of-function hotspots (74

273 \%) (Figure 5A). High-confidence driver genes supported by both overexpression and an

274 amplification peak: CCND1, CCND2, MYC, NSD2 (WHSC1), P2RY2/FCHSD2, FOXO3,

275 MAP3K14, CD40, LTBR, TNFRSF17, PRKCD, SEC62/SAMD7, SOX30 and GLI3

276 (Figure 5A). Of particular interest, TNFRSF 17 was involved by SVs in $5 \%$ of patients (n

$277=38)$ and encodes B-Cell Maturation Antigen (BCMA), a therapeutic target of chimeric

278 antigen receptor T-cells (CAR-T) and monoclonal antibodies (Figure 4B) ${ }^{41}$. We also

279 confirmed previous reports that virtually all SVs involving $M Y C$ resulted in its

280 overexpression, including deletions and inversions, which acted by repositioning MYC

281 next to the super-enhancers of NSMCE2, roughly $2 \mathrm{Mb}$ upstream $^{42}$. Numerous genes

282 were involved by gain-of-function hotspots without clear evidence of overexpression,

283 many of which are known to be important in multiple myeloma, such as $I R F 4, K L F 2$,

$284 \quad K L F 13$ and SLAMF7 (Table S4 and Figure 4C). Overall, translocation-type SVs

285 involving an immunoglobulin locus (i.e., $I G H, I G L$ or $I G K$ ), either directly or as part of a

286 complex event, were associated with the strongest gene expression effects (pairwise

287 Wilcoxon rank sum test, Bonferroni-Holm adjusted p-values $<0.0001)$ (Figure S5). This

288 was true both for canonical driver genes (median Z-score 1.7, IQR 1.1-2.4) and other

289 putative drivers (median Z-score 1.83, IQR 0.85-2.5). Non-immunoglobulin

290 translocations had the second highest impact, followed by tandem duplications and other

291 SVs, all of which were associated with significantly higher expression than patients

292 without SV (adjusted p-values < 0.01) (Figure S5).

293 Loss-of-function hotspots, defined by copy number loss and/or reduced

294 expression of a putative driver gene, were highly enriched for deletion breakpoints 
295 compared to the remaining hotspots (median 55 [IQR 40-68] \% vs 13 [7-23] \%;

296 Wilcoxon rank sum test $\mathrm{p}<0.001$; Table S4 and Figures 4D, 5A-B and S4). High-

297 confidence drivers defined by copy number loss and significantly reduced gene

298 expression were FAM46C, FAF1, RB1, MAX, SP140L, SCAF8, BIRC2 and CYLD

299 (Figure 5A). Additional genes identified to be associated with loss of copy number, but

300 without a clear effect on expression, included established tumor suppressor genes such as

301 TP53 and CDKN2C, as well as novel candidate drivers, e.g., SP3 and BCL2L11 (Table

302 S4). Some genes with particular enrichment of intragenic deletion breakpoints were large

303 and late-replicating, suggesting they may be fragile sites in multiple myeloma, prone to

304 breaking during replication stress (Figure 5C; Table S4 and S7) ${ }^{43}$. The top fragile gene

305 candidate in our data was PTPRD, which is suspected to be a tumor suppressor gene in

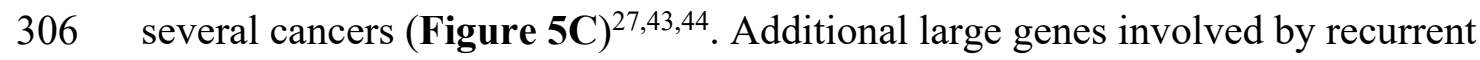

307 intragenic deletions in this study included CCSER1, FHIT and WWOX, all of which are

308 known fragile sites in cancer ${ }^{43,45-47}$. However, in a recent pan-cancer study, the

309 accumulation of complex nested deletion SVs (termed rigma) in fragile site-associated

310 genes including FHIT and $W W O X$ could not be fully explained by the classical features

311 of fragility, indicating that they may be positively selected ${ }^{6}$. Careful validation of the role

312 played by these hotspots will be necessary.

313 Consistent with recent findings in breast cancer ${ }^{37}$, in our series, SV hotspots

314 showed seven-fold enrichment for multiple myeloma germline predisposition SNPs as

315 compared with the remaining mappable genome (6 SNPs in $125 \mathrm{Mb}$ of hotspots vs 17 in

316 the remaining genome, Poisson test $p=0.0002$; Figure S6 $)^{48,49}$. The involved SNPs were

317 on 3q26.2 (SV hotspot involving SEC62/SAMD7), 6p21.3 (unknown SV target), 6q21 
318 (ATG5/PRDM1), 9p21.3 (CDKN2A/CDKN2B), 19p13.11 (KLF2) and 2q31.1 (SP3)

319 (Table S8). One additional SNP on 8q24.21 (CCAT1) was less than $1 \mathrm{Mb}$ upstream of

$320 M Y C$, between two SV hotspots centered around NSMCE2 and MYC, respectively.

321 Assuming that multiple myeloma predisposition SNPs and driver SVs affecting the same

322 locus would have similar consequences, this co-occurrence may provide novel insights

323 into the target genes and functional implications.

324 In our validation dataset of 52 genomes, 35 out of 100 hotspots showed

325 significant enrichment of SV breakpoints compared with the remaining mappable

326 genome (Poisson test, FDR $<0.1$; Table S9 and Figure S7A). Hotspots that were

327 confirmed in the validation dataset showed significantly higher prevalence in CoMMpass

328 as compared with hotspots that were not confirmed (median 4 [IQR 2.6-5.4] \% vs 2.2

$329 \quad[1.5-3] \%$; Wilcoxon rank sum test $\mathrm{p}<0.001)$.

330 Taken together, we identified 62 gain of function and 28 loss of function hotspots;

331 with 10 hotspots being of unknown function (Figures 5A, Table S4). Thirty-three

332 hotspots involved genes with established tumor suppressor or oncogene function in

333 multiple myeloma; 31 additional hotspots involved novel putative driver genes (Table

334 S4; all novel putative driver hotspots are shown in Figures 4B-C and S4). ${ }^{50}$

\section{Templated insertions are highly clustered and often involve multiple hotspots}

337 Templated insertion breakpoints were highly clustered across the genome (Figure

338 6A) and associated with copy number gain in $76 \%$ of cases (95\% CI 74-78 \%), but only

339 rarely with copy number loss $(8.3 \%$; $95 \%$ CI 7.0-9.6 \%). Gains were almost exclusively

340 single copy (92.5\% of 1200 gains), with less than one percent involving three or more 
341 copies above the baseline $(n=9)$, highlighting the stability of these events. Strikingly, out

342 of 449 templated insertion events, $73 \%$ involved one or more SV hotspots (including the

343 immunoglobulin loci) (Figure 6B-C). Half of the templated insertions $(\mathrm{n}=210)$ involved

344 more than two chromosomes and 45 (10\%) involved more than four chromosomes.

345 Among the 210 events spanning more than two chromosomes, $39 \%$ involved $M Y C$ or

$346 C C N D 1 ; 34 \%$ involved $I G H, I G L$ or $I G K$; and $30 \%$ involved none of the above, but one

347 or more other hotspots (Figure 6C). Overall, $54 \%$ of multi-chromosomal templated

348 insertions involved two or more hotspots (Figure 6B-C). In summary, we show that

349 templated insertions in multiple myeloma are highly clustered and often string together

350 amplified segments from multiple known drivers and SV hotspots in a single event.

352 Chromothripsis is associated with wide-spread gain and loss of gene function

353 mechanisms

354 In contrast to templated insertions, breakpoints of chromothripsis did not show

355 strong focal clustering (Figure 7A). Instead, running the PCF algorithm for

356 chromothripsis revealed enrichment in wider regions (Table S10). (Figure 7A). The

357 most commonly involved loci included a $32 \mathrm{Mb}$ region on chromosome $20 \mathrm{q} 11-13(\mathrm{n}=22$

358 patients involved); $40 \mathrm{Mb}$ spanning the $M Y C$ locus on 8q22-24 ( $\mathrm{n}=18) ; 23 \mathrm{Mb}$ on

$35917 q 12-23(n=16)$; and $12 \mathrm{Mb}$ on $12 \mathrm{p} 12-13(\mathrm{n}=16)$. An important feature of

360 chromothripsis is its ability to cause both gain- and loss-of-function as part of the same

361 event $^{51}$. Indeed, the breakpoints of chromothripsis were associated with chromosomal

362 loss in $50.1 \%$ of cases (95\% CI 48.8-52.5\%) and gain in 39.6 \% (95\% CI 37.7-41.5\%).

363 High-level focal copy number gains ( $>5$ copies) were predominantly caused by 
364 chromothripsis (75\% of segments) and present in only $6 \%$ of patients overall $(n=46)$

365 (Figure 7B-C). In contrast to solid tumors, where chromothripsis may result in double 366 minute chromosomes with $>50 \operatorname{copies}^{2,6,10,52}$, we observed no segments with more than 9

367 copies in this series. Similarly, in the validation cohort, the highest copy number

368 associated with chromothripsis was 7 (Figure S7B). Genes involved by high-level focal

369 gains had a two standard deviations higher expression, on average, as compared with the

370 same genes in patients with normal copy number (median Z-score 2.2 vs -0.12 , Wilcoxon

371 rank sum test, $\mathrm{p}<0.0001$ ) (Figure 7D). Interestingly, despite the profound overall impact

372 on gene expression, only four events (2.4\%) caused amplification and overexpression (Z-

373 score $>2$ ) of one or more established or putative drivers in multiple myeloma (i.e.,

$374 C C N D 1, C D 40, N F K B 1, M A P 3 K 14$ and $T N F R S F 17)^{13}$. For example, one patient

375 (MMRF_2330) had amplification and overexpression of MAP3K14 by chromothripsis,

376 along with multiple genes that have not been reported as genomic drivers in multiple

377 myeloma (Figure 7B). This included amplification and overexpression of the oncogene

$378 R A D 51 C$, which is commonly amplified in breast cancer and may play a drive role in this

379 one patient with multiple myeloma ${ }^{53}$. Two additional patients had $>6$ copies and outlier

380 overexpression of RPS6KB1, encoding the $70 \mathrm{kDa}$ ribosomal protein $\mathrm{S} 6$ kinase

381 (p70S6K), a crucial regulator of cell cycle, growth and survival in the PI3K/mTOR

382 pathways (Figure S7C) ${ }^{54}$. These observations raise questions regarding the impact of rare

383 SVs in deregulating potential drivers, and their contribution to multiple myeloma biology

384 overall.

386 Functional implications of recurrent and rare structural variants 
To determine the functional implications of rare SVs, we first identified all simple

388 and complex SVs where at least one breakpoint was potentially involved in a recurrent

389 event. SVs were assigned to categories of recurrent events in a hierarchical fashion, at

390 each step considering the SVs that had not yet been assigned. First, $9 \%$ of SVs were

391 assigned as canonical translocations, on the basis of one or more breakpoint involving an

392 immunoglobulin locus or a canonical partner gene; $6.2 \%$ were the cause of a known

393 recurrent CNA; $4.7 \%$ were involved an SV hotspot; and $17.4 \%$ had one or more

394 breakpoints within a wide GISTIC peak. Thirty-seven percent of all simple and complex

395 SVs had one or more breakpoint in a recurrently involved region, leaving $63 \%(\mathrm{n}=$

396 5577) as rare events (Figure 8A). Given the permissive criteria we used to define a

397 recurrent event, this is likely to be a conservative estimate of their true contribution.

398 There were striking differences in the proportion of rare and recurrent events between SV

399 classes: the vast majority of single tandem duplications, deletions and inversions were

400 rare, while $>75 \%$ of chromothripsis, chromoplexy and templated insertions involved at

401 least one recurrent region (Figure 8A-B). While this may be expected to some extent

402 from the number of breakpoints involved by each event, it also supports the notion that

403 complex events involve regions that are important in multiple myeloma and are therefore

404 positively selected. Considering the individual breakpoints of each complex event, a

405 greater proportion of chromothripsis breakpoints were rare as compared with templated

406 insertions (Figure 8B). Interestingly, $29 \%$ of complex events remained orphan.

407 Determining the biological implications of rare events is challenging because

408 genomic drivers are typically defined based on recurrence above some background level.

409 To overcome this challenge, we evaluated the impact of rare events in aggregate, asking 
410 the question whether genes near rare SVs were more or less likely to show outlier

411 expression levels than what would be expected if all rare SVs were passenger events ${ }^{55}$.

412 Outlier expression was defined for each gene as a z-score of $+/-2$. Consistent with the

413 heterogenous nature of multiple myeloma, each patient had a median of 124 genes

414 showing outlier expression (IQR 52-436), of which a median of 3 (IQR 1-6) and 2 (IQR

415 1-5) outliers were within $1 \mathrm{Mb}$ of recurrent and rare SV breakpoints, respectively (Figure

416 S8A). Conversely, each SV was associated with a median of 1 expression outlier (IQR 1-

417 2), similar for recurrent and rare events. Chromothripsis and templated insertions were

418 associated with more outliers overall (Figure S8B-C), consistent with the complexity of

419 these events, sometimes associated with dramatic effects on gene expression (as shown in

420 Figure 7D). Structural variants showed striking enrichment for outlier over- and under-

421 expression of nearby genes when compared with a permutation background model

422 (Methods). Recurrent and rare events showed similar overall trends, although the

423 magnitude of effect was greater for recurrent events (Figure 8C-D, respectively). The

424 directions of gene expression effects were consistent with the mechanism of each SV

425 class. Tandem duplications and templated insertions were strongly enriched for outlier

426 overexpression, with fewer nearby genes downregulated than expected by chance.

427 Tandem duplications exerted their effects only when involving the gene body; while

428 templated insertions maintained their effect up to $1 \mathrm{Mb}$ away from the gene, indicating a

429 major effect on gene regulatory mechanisms, e.g., super-enhancer hijacking.

430 Chromothripsis and unspecified complex events were associated with both up- and down-

431 regulation, through direct gene involvement as well as regulatory effects. 
433 estimate the number of outliers likely caused by SVs (Figure S8D). Recurrent templated

434 insertions and chromothripsis events were associated with a net excess of 1334 outliers

435 (95\% CI 1168-1490); while recurrent events of the other classes combined were

436 associated with an excess of 595 (95\% CI 416-759) outliers. For rare SVs, the

437 corresponding numbers were 197 (95\% CI 155-233) and 427 (95\% CI 299-547),

438 respectively. These numbers certainly underestimate the overall impact of SVs, as we

439 only considered direct effects on gene expression, applying a strict definition of outlier

440 expression. Although the specific target genes often remain elusive, these data

441 demonstrate that rare SVs strongly influence gene expression, which may contribute to

442 the biology of individual tumors.

\section{Discussion}

445 We describe the first comprehensive analysis of SVs in a large series of multiple 446 myeloma patients with paired whole genome and RNA sequencing. Our findings reveal

447 how simple and complex SVs shape the driver landscape of multiple myeloma, with

448 events ranging from common CNAs and canonical translocations to a large number of

449 non-recurrent SVs seemingly able to exert significant biological effects. The results focus

450 attention on the importance of SVs in multiple myeloma and on the use of whole genome

451 analyses in order to fully understand its driver landscape.

452 Previous studies of SVs in multiple myeloma have focused on translocations

453 without consideration of complex events ${ }^{14,38}$, and our previous WGS study of 30 patients

454 lacked the power to perform comprehensive driver discovery ${ }^{20}$. Here, applying a robust 
455 statistical approach ${ }^{37}$, we identified $100 \mathrm{SV}$ hotspots, 85 of which have not previously

456 been reported. Integrated analysis of copy number changes, gene expression and the

457 distribution of SV breakpoints revealed 31 new potential driver genes, including the

458 emerging drug targets TNFRSF17 (BCMA) and SLAMF7 ${ }^{41,56}$. Overall, our

459 comprehensive catalogue of SV hotspots expands the current understanding of driver

460 gene deregulation by recurrent CNAs and SVs and identified promising new driver

461 candidates.

462 From a pan-cancer perspective, the SV landscape of multiple myeloma is

463 characterized by a lower SV burden and less genomic complexity than in many solid

464 tumors ${ }^{2,6,11}$. For example, we did not find any classical double minute chromosomes with

465 tens to hundreds of amplified copies, nor did we find any of the recently proposed

466 complex SV classes pyrgo, rigma and tyfonas ${ }^{6}$. Nonetheless, we found that complex SVs

467 play a crucial role in shaping the genome of multiple myeloma patients. There are 3

468 major types of complex events seen in multiple myeloma, each of which is characterized

469 by distinct junctional features and distribution patterns. Chromothripsis breakpoints are

470 distributed across the genome in a chaotic fashion and are a common cause of recurrent

471 deletions and gains involving tumor suppressor genes and oncogenes. While

472 chromoplexy accounts for only a small proportion of SV breakpoints it emerged as an

473 important cause of chained events associated with deletions potentially inactivating a

474 range of tumor suppressor genes distributed across multiple chromosomes. In contrast,

475 templated insertions result in amplification and concatenation of multiple SV hotspots,

476 involving oncogenes and/or super-enhancers that may result in gene overexpression. The

477 frequency of templated insertions emphasizes the importance of enhancer hijacking in the 
478 pathogenesis of multiple myeloma and the relevance of transcriptional control in respect

479 to both its biology and therapy ${ }^{2,14}$.

480 A common feature of these 3 classes of SVs is the simultaneously deregulation of 481 multiple driver genes as part of a single event. Such multi-driver events are of particular

482 importance in myeloma progression as they can provide an explanation for the rapid

483 changes in clinical behavior that are frequently seen in the clinic ${ }^{57}$. Such changes may be

484 the result of "punctuated evolution" that occurs as a result of catastrophic intracellular

485 events leading to the complex SVs we describe.

486 Gene deregulation by SV is a major contributor to the biology of multiple

487 myeloma constituting a hallmark feature of its genome. However, the majority of SVs do

488 not involve known drivers, SV hotspots or regions of recurrent $\mathrm{CNV}$, implying they may

489 simply be passenger variants irrelevant to tumor biology. However, we show a

490 remarkable enrichment for gene expression outliers in proximity to rare SVs, suggesting

491 that rare SVs may make a significant contribution to the biological diversity of multiple

492 myeloma, including the mechanisms of disease progression and drug resistance.

493

494 Methods

$495 \quad$ Patients and samples

496 As a discovery cohort, we analyzed data from 762 patients with newly diagnosed

497 multiple myeloma enrolled in the CoMMpass study (NCT01454297). As a validation

498 cohort, we combined two multiple myeloma WGS datasets with a total of 52

499 patients $^{20,27,58}$. 
501 Whole genome sequencing and analysis

502 CoMMpass samples underwent low coverage long-insert WGS (median 4-8X).

503 Paired-end reads were aligned to the human reference genome (HRCh37) using the

504 Burrows Wheeler Aligner, BWA (v0.7.8). Somatic variant calling was performed using

505 Delly $(\mathrm{v} 0.7 .6)^{5}$ for SVs and tCoNuT for CNAs

506 (https://github.com/tgen/MMRF_CoMMpass/tree/master/tCoNut_COMMPASS). The

507 validation dataset was analyzed and comprehensively annotated as previously

508 described ${ }^{20,26}$.

RNA sequencing analysis and fusion calling

511 CoMMpass samples underwent RNA sequencing to a target coverage of 100

512 million reads. Paired-end reads were aligned to the human reference genome (HRCh37)

513 using STAR v2.3.1 $\mathrm{z}^{59}$. Transcript per million (TPM) gene expression values were

514 obtained using Salmon v7.2 ${ }^{60}$.

\section{Classification of structural variants}

517 Each pair of structural variant breakpoints (i.e. deletion, tandem duplication,

518 inversion or translocation) was classified as a single event, or as part of a complex event

519 (i.e. chromothripsis, chromoplexy or unspecified complex) ${ }^{2,20}$. Translocation-type events

520 were classified as single when involving no more than two breakpoint pairs and two

521 chromosomes, subdivided into reciprocal translocations, unbalanced translocations,

522 templated insertion or unspecified translocation as previously described ${ }^{2,20}$. Templated

523 insertions could be either simple or complex, depending on the number of breakpoints 
524 and chromosomes involved, but was always defined by translocations associated with

525 copy number gain. Chromothripsis was defined by more than 10 interconnected SV

526 breakpoint pairs associated with oscillating copy number across one or more

527 chromosomes. The thresholds of 10 breakpoints was imposed as a stringent criterion to

528 avoid overestimating the prevalence of chromothripsis. Chromoplexy was defined by $<10$

529 interconnected SV breakpoints across $>2$ chromosomes associated with copy number

530 loss. Patterns of three or more interconnected breakpoint pairs that did not fall into either

531 of the above categories were classified as unspecified "complex"20.

532

533 Mutational signature analysis

534 SNV calls from whole exome sequencing were subjected to mutational signature

535 fitting, using the previously described R package $m m s i g^{26}$. High APOBEC mutational

536 burden was defined by an absolute contribution of APOBEC mutations (mutational

537 signatures 2 and 13 ) in the $4^{\text {th }}$ quartile among patients with evidence of APOBEC

538 activity $^{26}$.

540 Structural basis for recurrent CNAs in multiple myeloma

541 We applied the following workflow to determine the structural basis for each

542 recurrent CNA in multiple myeloma (Table S2). First, we identified in each patient every

543 genomic segment involved by recurrent copy number gain or loss. Gains were defined by

544 total copy number $(\mathrm{CN})>2$; loss as a minor $\mathrm{CN}=0$. Second, we called whole arm events

545 in cases where the $\mathrm{CN}$ was equal to the $2 \mathrm{Mb}$ closest to the centromere and telomere, and

$546>90 \%$ of the arm had the same CN state. Third, for segments that did not involve the 
547 whole arm, we searched for SV breakpoints responsible for the CNA within $50 \mathrm{~kb}$ of the

548 CN segment ends. Finally, and intrachromosomal CNAs without SV support were

549 classified as unknown. We applied Fisher's exact test to compare the proportion of CNAs

550 explained by an SV for each of the recurrent events, requiring Bonferroni-Holm adjusted

551 p-values $<0.05$ for statistical significance.

552 Multi-driver events were defined by two or more independent driver copy number

553 segments and/or canonical driver translocations caused by the same SV (simple or

554 complex).

555

556 Copy number changes associated with structural variant breakpoints

557 To determine the genome-wide footprint of copy number changes resulting from

558 SVs, we employed an "SV-centric" workflow, as opposed to the CNA-centric workflow

559 described above. For each SV breakpoint, we searched for a change in copy number

560 within $50 \mathrm{~kb}$. If more than one CNA was identified, we selected the shortest segment.

561 Deletion and amplification CNAs were defined as changes from the baseline of that

562 chromosomal arm. As a baseline, we considered the average copy number of the two $\mathrm{Mb}$

563 closest to the telomere and centromere, respectively. This is important because deletions

564 are often preceded by large gains, particularly in patients with HRD. In those cases, we

565 are interested in the relative change caused by deletion, not the total $\mathrm{CN}$ of that segment

566 (which may still be $\geq 2$ ). We estimated the proportion of breakpoints associated with

567 copy number gain or loss across patients, collapsing the data in $2 \mathrm{Mb}$ bins across the

568 genome. Confidence intervals were estimated using bootstrapping and the quantile

569 method. For the purposes of plotting (Figures 4A, 6A and 7A), we divided the SV- 
570 associated CNAs into bins of $2 \mathrm{Mb}$. The resulting cumulative CNA plot shows the

571 number of patients with an SV-associated deletion or amplification.

\section{Hotspots of structural variation breakpoints}

$574 \quad$ To identify regions enriched for SV breakpoints, we employed the statistical

575 framework of piecewise constant fitting (PCF). In principle, the PCF algorithm identifies

576 regions where SVs are positively selected, based on enrichment of breakpoints with short

577 inter-breakpoint distance compared to the expected background. We used the

578 computational workflow previously described by ${ }^{37}$. In brief, negative binomial regression

579 was applied to model local SV breakpoint rates under the null hypothesis (i.e. absence of

580 selection), taking into account local features such as gene expression, replication time,

581 non-mapping bases and histone modifications. The PCF algorithm can define hotspots

582 without the use of binning, based on a user-defined smoothing parameter and threshold of

583 fold-enrichment compared to the background. This allows for hotspots of widely different

584 sized to be identified, depending on the underlying biological processes. To avoid calling

585 hotspots driven by highly clustered breakpoints in a few samples, we also set a minimum

586 threshold of 8 samples involved ( $\sim 1 \%$ of the cohort) to be considered hotspot, as

587 previously reported ${ }^{37}$. Despite this threshold, we found that complex SVs with tens to

588 hundreds of breakpoints in a localized cluster (particularly chromothripsis) came to

589 dominate the results. To account for this, we ran the PCF algorithm twice on different

590 subsets of the data: 1) considering all breakpoints of non-clustered SVs (simple classes

591 and templated insertions); and 2) including all SV classes, but randomly downsampling 
592 the data to include only one breakpoint per $500 \mathrm{~kb}$ per patient. Final output from both

593 approaches was merged for downstream analysis.

$594 \quad$ The full SV hotspot analysis workflow is attached as Data S1, drawing on generic

595 analysis tools that we have made available on github

596 (https://github.com/evenrus/hotspots/tree/hotornot-mm). The workflow is easily portable

597 to other datasets and includes utilities for data preparation and background model

598 generation as well as hotspot discovery and visualization.

600 Functional classification of structural variation hotspots

SV hotspots were classified based on local copy number and gene expression data

602 as gain of function, loss of function, or unknown.

604 applied the GISTIC v2.0 algorithm to identify wide peaks of enrichment for

605 chromosomal amplification or deletion (FDR $<0.1$ ), using standard settings ${ }^{39}$. Second,

606 we considered the cumulative copy number profiles of each hotspot, considering only the

607 patients with SV breakpoints within the region, looking for more subtle patterns of

608 recurrent CNA that was not picked up in the genome-wide analysis.

609 To determine the effects of SV hotspot involvement on gene expression, we

610 performed multivariate modeling using the limma package in $\mathrm{R}^{61}$. Gene expression count

611 data were first normalized using the DESeq/vst package, which allows the use of linear

612 models $^{62}$. We went on to estimate the effect SV hotspot involvement on every expressed

613 gene after adjustment for the primary molecular classes, $M Y C$ translocation and

614 gain1q21. Significantly altered expression (Bonferroni-Holm adjusted p-values $<0.01$ ) of 
615 a putative driver gene within $5 \mathrm{Mb}$ of the hotspot was considered as supporting evidence

616 for a driver role.

\section{Identification of putative driver genes involved by SV hotspots}

619 Multiple lines of evidence were considered to identify driver genes involved by

620 SV hotspots. Evidence of a putative driver gene included: 1) involved by driver SNVs in

621 multiple myeloma ${ }^{19,20} ; 2$ ) included in the COSMIC cancer gene census

622 (https://cancer.sanger.ac.uk/census); 3) designated as putative driver gene in The Cancer

623 Genome Atlas $\left.{ }^{63-66} ; 4\right)$ enrichment of SV breakpoints in or around the gene; 5) nearby

624 peak of SV-related copy number gain or loss; 6) SV classes and recurrent copy number

625 changes corresponding to a known role of that gene in cancer (i.e. oncogene or tumor

626 suppressor); and 7) differential gene expression. Having identified candidate driver genes

627 involved by SV hotspots, we reviewed the literature for evidence of a role in multiple

628 myeloma (Table S4).

630 Histone H3K27ac and super-enhancers

631 Active enhancer (H3K27ac) and super-enhancer data from primary multiple

632 myeloma cells was obtained from ${ }^{40}$. Enrichment of super-enhancers in hotspots was

633 assessed using a Poisson test, comparing the super-enhancer density within $100 \mathrm{~kb}$ of

634 hotspots with the remaining mappable genome.

635

636 Enrichment of germline predisposition loci 
Germline predisposition SNPs for multiple myeloma were obtained from a recent

639 performed as for super-enhancers. We also simulated 1000 sets of hotspots of identical

640 size using a bed shuffle implementation in $\mathrm{R}^{67}$, estimating the probability under the null

641 hypothesis to obtain a more extreme result than the observed data. As a final control

642 measure, we repeated the same analysis for loci associated with development of a

643 completely unrelated condition. We selected schizophrenia as a control condition because

644 the pathogenesis is distinct from multiple myeloma, with abundant data on germline risk

$645 \operatorname{loci}^{68}$.

\section{External validation of hotspots}

648 Hotspots identified by the PCF algorithm were validated in our external cohort of

64952 patients. We applied one-sided poisson tests for enrichment of SV breakpoints within

650 each defined hotspot region as compared with the mappable non-hotspot genome (FDR <

651 0.1). To avoid bias from highly clustered events, we first downsampled the data as

652 described for the main hotspot analysis, randomly selecting a single breakpoint per 500

$653 \mathrm{~kb}$ per patient.

\section{Chromothripsis hotspot analysis}

656 Empirical background models showed very poor ability to predict the distribution

657 of chromothripsis breakpoints, as may be expected if DNA breaks in chromothripsis tend

658 to be random. To identify regions enriched for chromothripsis, we applied the PCF

659 algorithm with a uniform background, only adjusting for non-mapping bases. 


\section{Gene expression analysis of rare structural variants}

To determine whether rare SVs are functionally relevant, we assessed their

663 aggregated effect on gene expression outliers, adopting an approach previously published

664 for germline $\mathrm{SVs}^{55}$. First, we selected all genes in our cohort requiring $>0$ TPM

665 expression in $>25 \%$ of patients and a median expression level of $>1$. Second, we defined

666 expression outliers with a Z-score of $+/-2$ for each gene. Genes involved by SVs was

667 defined separately for deletion/tandem duplication type SVs and translocation/inversion

668 types. For deletions and tandem duplications, only focal events were considered (size $<3$

$669 \mathrm{Mb}$ ), and genes were considered directly involved if the gene was between the

670 breakpoints, or if a breakpoint transected the gene. Genes within $100 \mathrm{~kb}$ or $100 \mathrm{~kb}-1 \mathrm{Mb}$

671 of a breakpoint were considered as separate categories. For translocations and inversions,

672 direct involvement was considered when the breakpoint transected the gene. Genes

673 within $100 \mathrm{~Kb}$ and $100 \mathrm{~Kb}-1 \mathrm{Mb}$ were considered as separate categories. Having

674 defined gene expression outliers and genes involved by SVs, we compared the observed

675 number of outliers within each category with the numbers expected by chance. In the

676 background permutation model, we randomly shuffled the outlier status (up, down or

677 non-outlier) of each gene. The median frequency across permutations was used as

678 background estimates, with $95 \%$ confidence intervals estimated by the quantile method.

679 Fold enrichment and net excess of outliers was based on the observed frequency divided

680 by the background frequency and the upper and lower bounds of its $95 \%$ confidence

681 interval. 


\section{Data and software availability}

683 All the raw data used in the study are already publicly available (dbGap:

684 phs000748.v1.p1, dbGap: phs000348.v2.p1 and European Genome phenome Archive:

685 EGAD00001001898. Analysis was carried out in R version 3.6.1. The full analytical

686 workflow in R to identify hotspots of structural variants is provided in Data S1. All other

687 software tools used are publicly available. Analysis code will be provided by the Lead

688 Contact upon request.

690 Acknowledgements

691 This work is supported by the Memorial Sloan Kettering Cancer Center NCI Core Grant

692 (P30 CA 008748), the Multiple Myeloma Research Foundation (MMRF), and the

693 Perelman Family Foundation.

694 K.H.M. is supported by the Haematology Society of Australia and New Zealand New

695 Investigator Scholarship and the Royal College of Pathologists of Australasia Mike and

696 Carole Ralston Travelling Fellowship Award.

697 G.J.M is supported by The Leukemia Lymphoma Society.

\section{Author contributions}

700 F.M. designed and supervised the study, collected and analyzed data and wrote the paper.

701 E.H.R. designed the study, collected and analyzed data and wrote the paper. G.J.M. and

702 O.L. collected and analyzed data and wrote paper. D.G., V.Y, K.M. and B.D.D, analyzed

703 data and wrote the paper. P.J.C., E.P., G.G., D.L. and N.A., analyzed data. E.M.B., C.A., 
bioRxiv preprint doi: https://doi.org/10.1101/2019.12.18.881086; this version posted December 19, 2019. The copyright holder for this preprint (which was not certified by peer review) is the author/funder. All rights reserved. No reuse allowed without permission.

704 M.H., A.D., Y.Z., P.B., D.A., K.C.A., P.M., N.B., H.A.L., N.M., J.K., G.M., collected

705 data.

706

707 Declarations of interests

708 No conflict of interests to declare.

709 


\section{$710 \quad$ Figure legends}

\section{Figure 1: Simple and complex classes of structural variants in multiple myeloma. A:}

712 Genome-wide burden of structural variant types (horizontal panels) in each patient,

713 grouped according to primary multiple myeloma molecular subgroup. Bars within each

714 panel are colored according to the contribution of complex SV classes (i.e. chromoplexy,

715 chromothripsis, templated insertions or other 'complex' variants grouped together) versus

716 simple SVs (DEL, deletion; DUP, tandem duplication; INV, inversion and TRA,

717 translocation). Heatmap below shows the presence of complex structural variant classes

718 together with key genomic drivers of multiple myeloma, including non-canonical

719 immunoglobulin (IG) translocations. B-C: Kaplan-meier plots for progression free

720 survival (PFS) (B) and overall survival (OS) (C) in patients with and without

721 chromothripsis (shown in blue and red respectively). D: Hazard ratio for PFS and OS by

722 SV type, estimated using multivariate Cox regression. (Line indicates $95 \%$ CI from

723 multivariate cox regression models, statistically significant features indicated by asterisks

$724 \quad(* \mathrm{p}<0.05 ; * * \mathrm{p}<0.01)$. For templated insertions, chromothripsis, chromoplexy and

725 unspecified complex events, we compared patients with 0 versus 1 or more events. The

726 remaining SVs were considered by their simple class (i.e. DUP, DEL, TRA and INV),

727 comparing the $4^{\text {th }}$ quartile SV burden with the lower three quartiles. The multivariate

728 models included all SV variables as well the following clinical and molecular features:

729 age, sex, ECOG status, ISS-stage, induction regimen, gain1q21, delFAM46C, delTRAF3,

730 delTP53, delRB1, high APOBEC mutational burden, hyperdiploidy and canonical

731 translocations involving CCND1, MMSET, MAF, MAFA, MAFB and MYC. 


\section{Figure 2: Structural basis of recurrent translocations and copy number changes. A:}

734 Relative contribution (y-axis) of simple and complex SV classes to translocations (TRA)

735 involving the immunoglobulin loci and $M Y C$ with canonical and non-canonical partners

736 (x-axis). The category "Other" contains non-MYC translocations for $I G K$ and $I G L$, while

737 for $I G H$ it includes $C C N D 2, C C N D 3, M A F A$ and $M A F B$. B: Transcript per million

738 (TPM) gene expression of canonical and non-canonical partners of translocations

739 involving the immunoglobulin (IG) loci. Each point represents a sample, colored by the

740 SV class involved or absence of SV (gray). Boxplots shows the median and interquartile

741 range (IQR) of expression across all patients, with whiskers extending to $1.5 * \mathrm{IQR}$. The

742 templated insertion of $I G H$ and $M A F$ with low expression was part of a multi-

743 chromosomal event involving and causing the overexpression of $C C N D 1$. C) Structural

744 basis of recurrent multiple myeloma CNA drivers, showing the relative contribution of

745 whole arm events and CNAs associated with a specific SV. Intrachromosomal events

746 without a clear causal SV were classified as "unknown”. D) Co-occurrence matrix

747 showing all cases where two or more recurrent CNAs and/or canonical driver

748 translocations were caused by the same simple or complex SV. Each CNA segment was

749 only counted once, even if including more than one driver gene. For example, a deletion

750 involving both $C D K N 2 C$ and FAM46C will be reported only if connected to another

751 CNA caused by the same SV. BIRC: BIRC2/BIRC3.

753 Figure 3: Multiple recurrent aberrations caused by the same SV. A-C) Genome plots

754 showing the SV breakpoints of a single complex SV in each panel (colored lines), with

755 bars around the plot circumference indicating copy number changes. The outer track 
756 represents copy number loss (red), the inner represents copy number gains (blue). A)

757 Chromothripsis involving $I G H$ and 9 recurrent driver aberrations across 10 different

758 chromosomes (sample MMRF_1890_1_BM). B) Chromoplexy involving chromosomes

75911,13 , and 14, simultaneously causing deletion of key tumor suppressor genes on each

760 chromosome (sample MMRF_2194_1_BM). C) Templated insertion involving 5

761 different chromosomes, causing a canonical IGH-CCNDI translocation and involving

762 MYC in the same chain of events. Focal amplifications can be seen corresponding to the

763 translocation breakpoints (sample MMRF_1862_1_BM). D) Top: Unbalanced inversion

764 on chromosome 6 , simultaneously causing gain of $6 \mathrm{p}$ and loss of $6 \mathrm{q}$ (sample

765 MMRF_1740_1_BM). Bottom: Copy number profile of chromosome 6 in sample

766 MMRF_2266_1_BM, generated through several independent structural variants. The

767 main events were: 1) whole chromosome gain; 2) unbalanced inversion causing an

768 additional gain of $6 \mathrm{p}$ and loss of one of the two duplicated 6q alleles; and 3) chromoplexy

769 involving chromosome 14 and 15, with loss of an interstitial segment containing SCAF8

770 and $A R I D 1 B$ on $6 \mathrm{q}$. This patient also had an independent chained translocation event

771 involving the $T X N D C 5 / B M P 6$ locus on $6 \mathrm{p}$.

\section{Figure 4: Genome-wide distribution of structural variation breakpoints and}

774 hotspots. A) Top: Rainfall plot showing the log-distance of each SV breakpoint (points)

775 to its closest neighbor (y-axis) across the genome (x-axis), colored by SV class (legend

776 above figure). Middle: Distribution of SV hotspots (green) and recurrent copy number

777 changes (red/blue) identified by the GISTIC algorithm. Bottom: all copy number changes

778 caused by SV breakpoints, showing cumulative plots for gains (blue) and losses (red). B- 
779 D) Zooming in on three SV hotspots, showing the chromosomal region (top), cumulative

780 copy number and GISTIC peaks (middle), and the breakpoint density of relevant SV

781 classes (colors indicated in legend above A) around the hotspot (bottom). The lower plots

782 are annotated with enhancer marks (histone H3K27ac) in brown and genes in gray, with

783 putative driver gene names in black. B) Gain of function hotspot centered around

784 TNFRSF17 (BCMA), dominated by highly clustered templated insertions, associated

785 with focal copy number gain of TNFRSF 17. C) Gain of function hotspot involving four

786 genes in the Signaling Lymphocyte Activation Molecule (SLAM) family of

787 immunomodulatory receptors, including the gene encoding the monoclonal antibody

788 target SLAMF7. The SV hotspot region was supported by a GISTIC peak of copy number

789 gain. D) Deletion hotspot associated with copy number loss centered on the cyclin

790 dependent kinase inhibitors $C D K N 2 A / C D K N 2 B$, supported by a peak of copy number

791 loss identified by GISTIC.

792

793 Figure 5: Gain- and loss- of function consequences of structural variant hotspots. A)

794 Summary of all 100 SV hotspots, showing (from the top): absolute and relative

795 contribution of SV classes within $100 \mathrm{~Kb}$ of the hotspot; involvement of active enhancers

796 in multiple myeloma, copy number changes and differential expression of putative driver

797 genes; known and candidate driver genes. B) Distribution of SV classes involving each

798 hotspot, colored by gain-of-function (green), loss-of-function (orange) or unknown

799 functional status (blue) as defined by copy number and gene expression data. Percentage

800 of templated insertion, simple translocation and tandem duplication SVs on the y-axis

801 and deletion SVs x-axis. C) Potential fragile sites in multiple myeloma, characterized by 
802 deletions (y-axis) within the body of large genes (size) that are late replicating (x-axis).

803 Fragile sites indicated by the color scale have been validated in pan-cancer studies.

805 Figure 6: Templates insertions are highly clustered and string together multiple

806 hotspots. A) Distribution of templated insertions across the genome (above) and

807 associated cumulative copy number changes (below). Vertical black lines indicate SV

808 hotspots containing $>3$ patients with a templated insertion. Selected gene names are

809 annotated. Numbers are annotated where peaks extend outside of the plotting area B) Co-

810 occurrence matrix showing the number of cases where pairs of SV hotspots occur as part

811 of the same templated insertion, involving two or more chromosomes. C) Example of a

812 templated insertion chain (brown lines) spanning three SV hotspots, involving the $I G L$,

$813 M Y C$, and a hotspot on chromosome 15q24 (sample MMRF_1550_1_BM). Copy number

814 profile shown in blue, with an active enhancer track below (H3K27Ac).

816 Figure 7: Wide-spread involvement of chromothripsis with gain and loss of function

817 effects. A) Distribution of chromothripsis involvement across the genome (above), with

818 associated cumulative copy number changes (below). Black lines indicate hotspots of

819 chromothripsis. B) Example of chromothripsis causing high-level focal gains on

820 chromosome 17 (sample MMRF_2330_1_BM). The horizontal black line indicates total

821 copy number; the dashed orange line minor copy number. Vertical lines represent SV

822 breakpoints, color-coded by SV class. Selected overexpressed genes (Z-score $>2)$ are

823 annotated in red, including the established multiple myeloma driver gene $M A P 3 K 14$, and

$824 R A D 51 C$, an oncogene commonly amplified in breast cancer (6 copies). C) Number of 
825 focal segments with $>5$ copies across chromosomes, colored by SV class. D) Violin plot

826 showing gene expression Z-scores of genes involved by high-level gains ( $>5$ copies)

827 compared with expression of the same genes in patients where they have normal copy

828 number. Points with error bars show median and interquartile range.

830 Figure 8: Distribution and impact of recurrent and rare structural variations. A)

831 Proportion of single and complex SVs involved in one or more known or novel region of

832 recurrent SV and/or CNA. Canonical translocation loci were prioritized (i.e. $I G H, I G K$,

$833 I G L, M Y C, C C N D 1, C C N D 2, C C N D 3, M A F, M A F A, M A F B$ and MMSET), followed by

834 known recurrent aneuploidies, SV hotspots and GISTIC peaks (See also Tables S5-S6).

835 The leftover category "rare SV" constitutes those events where every breakpoint fell

836 outside of the above-mentioned recurrently involved regions. For example, a

837 chromothripsis event with 100 breakpoints will be classified as "Canonical TRA" if one

838 breakpoint falls within the $I G H$ locus, even if the remaining 99 breakpoints are in non-

839 recurrent regions. B) Heatmaps showing the proportion of breakpoints falling into each of

840 the categories in A), displaying chromothripsis events (above) and templated insertion

841 event involving more than two chromosomes (below). Each column in the heatmaps

842 represent one complex event, arranged and color-coded (above the heatmap) according to

843 the classification in A). Rows in the heatmap show the proportion of breakpoints

844 belonging to each complex event that fall into each of the categories, e.g., Canonical

845 TRA, Recurrent CNA, etc. The sum of proportions may exceed 1 for each event, because

846 many of the recurrent events overlap, e.g. the same region may be both recurrently

847 translocated, an SV hotspot, and a GISTIC peak. Breakpoints falling in that region will 
848 be counted towards each of the categories. C-D) Enrichment analysis of outlier gene

849 expression (Z-score $+/-2$ ) by proximity to recurrent (C) and rare (D) SVs, showing the

$850 \log 10$ fold-enrichment (y-axis) for each SV class (x-axis), compared with a permutation-

851 based background model. Enrichment for overexpression outliers above and outlier

852 reduced expression below. Columns show outlier enrichment according to the distance

853 from SV breakpoints to involved genes.

854 


\section{Supplementary materials}

856 Table S1: Cohort summary. Baseline clinical variables and availability of sequencing

857 data from patients in the CoMMpass cohort.

858

859 Table S2: Recurrent CNAs in multiple myeloma. Chromosomal coordinates used to

860 define recurrent CNAs in multiple myeloma.

861

862 Table S3: Proportion of CNAs explained by an SV in CoMMpass and the validation

863 dataset. For each of the recurrent CNAs in multiple myeloma, we report the proportion

864 of events explained by an SV in the CoMMpass and the validation dataset. Odds ratios

865 (OR) are from Fisher's exact test, with Bonferroni-Holm adjusted p-values.

866

867 Table S4: SV hotspots in multiple myeloma. Summary table of all SV hotspots.

868 Detailed explanations for each column are provided as a second sheet in the table excel 869 file.

870

871 Table S5: GISTIC amplification peaks. Wide peaks of chromosomal gains as defined

872 by the GISTIC algorithm (FDR $<0.1)$.

873

874 Table S6: GISTIC deletion peaks. Wide peaks of chromosomal loss as defined by the

875 GISTIC algorithm (FDR $<0.1)$. 
877 Table S7: Evaluation of fragile sites in multiple myeloma. Shows all genes with three

878 or more patients harboring deletion breakpoints within the gene body. Genes are assigned

879 as known fragile sites if their genomic coordinates intersect with previously reported

880 fragile sites in cancer, or as potential fragile sites if their size exceeds $500 \mathrm{~Kb}$ and they

881 are late replicating. Late replicating was defined here as below the mean (i.e. normalized

882 replication time below zero) across the genome of lymphoblastoid cells.

\section{Table S8: Germline predisposition loci in multiple myeloma and associated SV}

885 hotspots. Shows all previously described germline predisposition loci for multiple

886 myeloma, including the genes involved (SNP_gene) and suspected target genes

887 (SNP_target), as previously reported. We go on to report the overlapping SV hotspot (see 888 also Table S4).

890 Table S9: Validation of SV hotspots. Number and proportion of patients with an SV in

891 each hotspot across CoMMpass and the validation dataset, with results from Poisson-tests

892 for hotspot enrichment in the validation dataset.

894 Table S10: Hotspots of chromothripsis. Hotspot regions for chromothripsis identified

895 by the PCF algorithm when run exclusively with chromothripsis breakpoints. 


\section{References}

8981 Zhang, Y. et al. A Pan-Cancer Compendium of Genes Deregulated by Somatic

899 Genomic Rearrangement across More Than 1,400 Cases. Cell Rep 24, 515-527, 900 doi:10.1016/j.celrep.2018.06.025 (2018).

9012 Li, Y. et al. Patterns of structural variation in human cancer. bioRxiv, 181339, 902 doi:10.1101/181339 (2017).

9033 Yang, L. et al. Diverse Mechanisms of Somatic Structural Variations in Human Cancer Genomes. Cell 153, 919-929,

905 doi:https://doi.org/10.1016/j.cell.2013.04.010(2013).

9064 Maciejowski, J. \& Imielinski, M. Modeling cancer rearrangement landscapes.

907 Current Opinion in Systems Biology 1, 54-61,

908 doi:https://doi.org/10.1016/j.coisb.2016.12.005 (2017).

9095 Rausch, T. et al. DELLY: structural variant discovery by integrated paired-end

910 and split-read analysis. Bioinformatics 28, i333-i339,

911 doi:10.1093/bioinformatics/bts378 (2012).

9126 Hadi, K. et al. Novel patterns of complex structural variation revealed across

913 thousands of cancer genome graphs. bioRxiv, 836296, doi:10.1101/836296

914 (2019).

9157 Mitchell, T. J. et al. Timing the Landmark Events in the Evolution of Clear Cell

916 Renal Cell Cancer: TRACERx Renal. Cell 173, 611-623.e617,

917 doi:10.1016/j.cell.2018.02.020 (2018).

9188 Glodzik, D. et al. Mutational mechanisms of amplifications revealed by analysis

919 of clustered rearrangements in breast cancers. Annals of oncology : official

920 journal of the European Society for Medical Oncology / ESMO 29, 2223-2231,

921 doi:10.1093/annonc/mdy404 (2018).

9229 Lee, J. J. et al. Tracing Oncogene Rearrangements in the Mutational History of Lung Adenocarcinoma. Cell 177, 1842-1857.e1821,

924 doi:10.1016/j.cell.2019.05.013 (2019).

92510 Zhang, C.-Z. et al. Chromothripsis from DNA damage in micronuclei. Nature

926 522, 179-184, doi:10.1038/nature14493 (2015).

92711 Wala, J. A. et al. Selective and mechanistic sources of recurrent rearrangements 928 across the cancer genome. bioRxiv, 187609, doi:10.1101/187609 (2017).

92912 Maciejowski, J., Li, Y., Bosco, N., Campbell, P. J. \& de Lange, T.

930 Chromothripsis and Kataegis Induced by Telomere Crisis. Cell 163, 1641-1654, 931 doi:10.1016/j.cell.2015.11.054 (2015). 
93213 Manier, S. et al. Genomic complexity of multiple myeloma and its clinical

933 implications. Nat Rev Clin Oncol 14, 100-113, doi:10.1038/nrclinonc.2016.122

934 (2017).

93514 Barwick, B. G. et al. Multiple myeloma immunoglobulin lambda translocations

$936 \quad$ portend poor prognosis. Nature communications 10, 1911, doi:10.1038/s41467-

937 019-09555-6 (2019).

93815 Bolli, N. et al. Genomic patterns of progression in smoldering multiple myeloma.

939 Nature communications 9, 3363, doi:10.1038/s41467-018-05058-y (2018).

$94016 \quad$ Misund, K. et al. MYC dysregulation in the progression of multiple myeloma.

941 Leukemia, doi:10.1038/s41375-019-0543-4 (2019).

94217 Walker, B. A. et al. Translocations at 8q24 juxtapose MYC with genes that harbor superenhancers resulting in overexpression and poor prognosis in myeloma patients. Blood Cancer J 4, e191, doi:10.1038/bcj.2014.13 (2014).

94518 Walker, B. A. et al. A compendium of myeloma-associated chromosomal copy number abnormalities and their prognostic value. Blood 116, e56-e65,

94819 Walker, B. A. et al. Identification of novel mutational drivers reveals oncogene dependencies in multiple myeloma. Blood 132, 587-597, doi:10.1182/blood-201803-840132 (2018).

20 Maura, F. et al. Genomic landscape and chronological reconstruction of driver

21 Walker, B. A. et al. A high-risk, Double-Hit, group of newly diagnosed myeloma events in multiple myeloma. Nature communications 10, 3835, doi:10.1038/s41467-019-11680-1 (2019). identified by genomic analysis. Leukemia 33, 159-170, doi:10.1038/s41375-018-

95722 Weinhold, N. et al. Clonal selection and double-hit events involving tumor

96023 Jones, J. R. et al. Clonal evolution in myeloma: the impact of maintenance

96424 Korbel, J. O. \& Campbell, P. J. Criteria for inference of chromothripsis in cancer suppressor genes underlie relapse in myeloma. Blood 128, 1735-1744, doi:10.1182/blood-2016-06-723007 (2016).

96625 Baca, S. C. et al. Punctuated evolution of prostate cancer genomes. Cell 153, 666967 677, doi:10.1016/j.cell.2013.03.021 (2013). 
96826 Rustad, E. H. et al. Timing the Initiation of Multiple Myeloma. Sneak Peak (pre-

969 print), doi:https://dx.doi.org/10.2139/ssrn.3409453 (2019).

97027 Lohr, J. G. et al. Widespread genetic heterogeneity in multiple myeloma:

971

972 implications for targeted therapy. Cancer Cell 25, 91-101,

97328 Maura, F. et al. Biological and prognostic impact of APOBEC-induced mutations in the spectrum of plasma cell dyscrasias and multiple myeloma cell lines.

975

976

29 Walker, B. A. et al. APOBEC family mutational signatures are associated with poor prognosis translocations in multiple myeloma. Nature communications $\mathbf{6}$, 6997, doi:10.1038/ncomms7997 (2015).

980

30 Magrangeas, F., Avet-Loiseau, H., Munshi, N. C. \& Minvielle, S. Chromothripsis

981

982 identifies a rare and aggressive entity among newly diagnosed multiple myeloma patients. Blood 118, 675-678, doi:10.1182/blood-2011-03-344069 (2011).

983

984

32 Quigley, D. A. et al. Genomic Hallmarks and Structural Variation in Metastatic Prostate Cancer. Cell 174, 758-769.e759, doi:10.1016/j.cell.2018.06.039 (2018).

Rausch, T. et al. Genome sequencing of pediatric medulloblastoma links catastrophic DNA rearrangements with TP53 mutations. Cell 148, 59-71, doi:10.1016/j.cell.2011.12.013 (2012).

35 Boothby, M. R., Hodges, E. \& Thomas, J. W. Molecular regulation of peripheral

Grobner, S. N. et al. The landscape of genomic alterations across childhood cancers. Nature 555, 321-327, doi:10.1038/nature25480 (2018). antibody-secreting plasma cells. Nat Rev Immunol 15, 160-171, doi:10.1038/nri3795 (2015).

99937 Glodzik, D. et al. A somatic-mutational process recurrently duplicates germline susceptibility loci and tissue-specific super-enhancers in breast cancers. Nat Genet 1001 49, 341-348, doi:10.1038/ng.3771 (2017). 
100238 Walker, B. A. et al. Characterization of IGH locus breakpoints in multiple

1003

1004

1005

1006

1007

1008

1009

1010

1011

1012

1013

1014

1015

1016

1017

1018

1019

1020

1021

1022

1023

1024

1025

1026

1029

1030

1031

1032

1033

1034

1035

1036 myeloma indicates a subset of translocations appear to occur in pregerminal center B cells. Blood 121, 3413-3419, doi:10.1182/blood-2012-12-471888 (2013).

39 Mermel, C. H. et al. GISTIC2.0 facilitates sensitive and confident localization of the targets of focal somatic copy-number alteration in human cancers. Genome Biology 12, R41, doi:10.1186/gb-2011-12-4-r41 (2011).

40 Jin, Y. et al. Active enhancer and chromatin accessibility landscapes chart the regulatory network of primary multiple myeloma. Blood 131, 2138-2150, doi:10.1182/blood-2017-09-808063 (2018).

41 Cho, S. F., Anderson, K. C. \& Tai, Y. T. Targeting B Cell Maturation Antigen (BCMA) in Multiple Myeloma: Potential Uses of BCMA-Based Immunotherapy. Frontiers in immunology 9, 1821, doi:10.3389/fimmu.2018.01821 (2018).

42 Affer, M. et al. Promiscuous MYC locus rearrangements hijack enhancers but mostly super-enhancers to dysregulate MYC expression in multiple myeloma. Leukemia 28, 1725-1735, doi:10.1038/leu.2014.70 (2014).

43 Glover, T. W., Wilson, T. E. \& Arlt, M. F. Fragile sites in cancer: more than meets the eye. Nature reviews. Cancer 17, 489-501, doi:10.1038/nrc.2017.52 (2017).

44 Veeriah, S. et al. The tyrosine phosphatase PTPRD is a tumor suppressor that is frequently inactivated and mutated in glioblastoma and other human cancers. Proceedings of the National Academy of Sciences 106, 9435, doi:10.1073/pnas.0900571106 (2009).

45 Hussain, T., Liu, B., Shrock, M. S., Williams, T. \& Aldaz, C. M. WWOX, the FRA16D gene: A target of and a contributor to genomic instability. Genes, chromosomes \& cancer 58, 324-338, doi:10.1002/gcc.22693 (2019).

6 Patel, K. et al. FAM190A deficiency creates a cell division defect. The American journal of pathology 183, 296-303, doi:10.1016/j.ajpath.2013.03.020 (2013).

47 Waters, C. E., Saldivar, J. C., Hosseini, S. A. \& Huebner, K. The FHIT gene product: tumor suppressor and genome "caretaker". Cell Mol Life Sci 71, 45774587, doi:10.1007/s00018-014-1722-0 (2014).

48 Went, M. et al. Identification of multiple risk loci and regulatory mechanisms influencing susceptibility to multiple myeloma. Nature communications $\mathbf{9}, 3707$, doi:10.1038/s41467-018-04989-w (2018).

49 Janz, S. et al. Germline Risk Contribution to Genomic Instability in Multiple Myeloma. Frontiers in genetics 10, doi:10.3389/fgene.2019.00424 (2019). 
103750 Hu, G., Wei, Y. \& Kang, Y. The Multifaceted Role of MTDH/AEG-1 in Cancer

$1038 \quad$ Progression. Clinical Cancer Research 15, 5615, doi:10.1158/1078-0432.CCR-

1039 09-0049 (2009).

$104051 \quad$ Cortés-Ciriano, I. et al. Comprehensive analysis of chromothripsis in 2,658

1041

1042

$104352 \quad$ Stephens, P. J. et al. Massive Genomic Rearrangement Acquired in a Single

1044 human cancers using whole-genome sequencing. bioRxiv, 333617, doi:10.1101/333617 (2018).

1045

1046

Catastrophic Event during Cancer Development. Cell 144, 27-40, doi:10.1016/j.cell.2010.11.055 (2011).

Barlund, M. et al. Multiple genes at 17q23 undergo amplification and overexpression in breast cancer. Cancer research 60, 5340-5344 (2000). 67, 1019-1025, doi:10.1136/jclinpath-2014-202560 (2014).

55 Chiang, C. et al. The impact of structural variation on human gene expression. Nature Genetics 49, 692, doi:10.1038/ng.3834 (2017). and Clinical Activity of the Therapeutic SLAMF7 Antibody, Elotuzumab in Multiple Myeloma. Frontiers in immunology 9, 2551, doi:10.3389/fimmu.2018.02551 (2018). doi:10.1001/jamaoncol.2019.4659 (2019).

1061

Chapman, M. A. et al. Initial genome sequencing and analysis of multiple myeloma. Nature 471, 467-472, doi:10.1038/nature09837 (2011).

$106460 \quad$ Patro, R., Duggal, G., Love, M. I., Irizarry, R. A. \& Kingsford, C. Salmon provides fast and bias-aware quantification of transcript expression. Nature 
107363 Sanchez-Vega, F. et al. Oncogenic Signaling Pathways in The Cancer Genome

$1074 \quad$ Atlas. Cell 173, 321-337.e310, doi:10.1016/j.cell.2018.03.035 (2018).

107564 Seiler, M. et al. Somatic Mutational Landscape of Splicing Factor Genes and

1076 Their Functional Consequences across 33 Cancer Types. Cell Reports 23, 282-

1077 296.e284, doi:https://doi.org/10.1016/j.celrep.2018.01.088 (2018).

107865 Ge, Z. et al. Integrated Genomic Analysis of the Ubiquitin Pathway across Cancer 1079 Types. Cell Reports 23, 213-226.e213,

1080 doi:https://doi.org/10.1016/j.celrep.2018.03.047 (2018).

$108166 \quad$ Knijnenburg, T. A. et al. Genomic and Molecular Landscape of DNA Damage

1082 Repair Deficiency across The Cancer Genome Atlas. Cell Reports 23, 239-

1083 254.e236, doi:https://doi.org/10.1016/j.celrep.2018.03.076 (2018).

108467 Riemondy, K. A. et al. valr: Reproducible genome interval analysis in R.

1085 F1000Research 6, 1025-1025, doi:10.12688/f1000research.11997.1 (2017).

108668 Huo, Y., Li, S., Liu, J., Li, X. \& Luo, X.-J. Functional genomics reveal gene

1087 regulatory mechanisms underlying schizophrenia risk. Nature communications

1088 10, 670, doi:10.1038/s41467-019-08666-4 (2019). 
Copy number $\square$ Gain $\square$ Loss

A

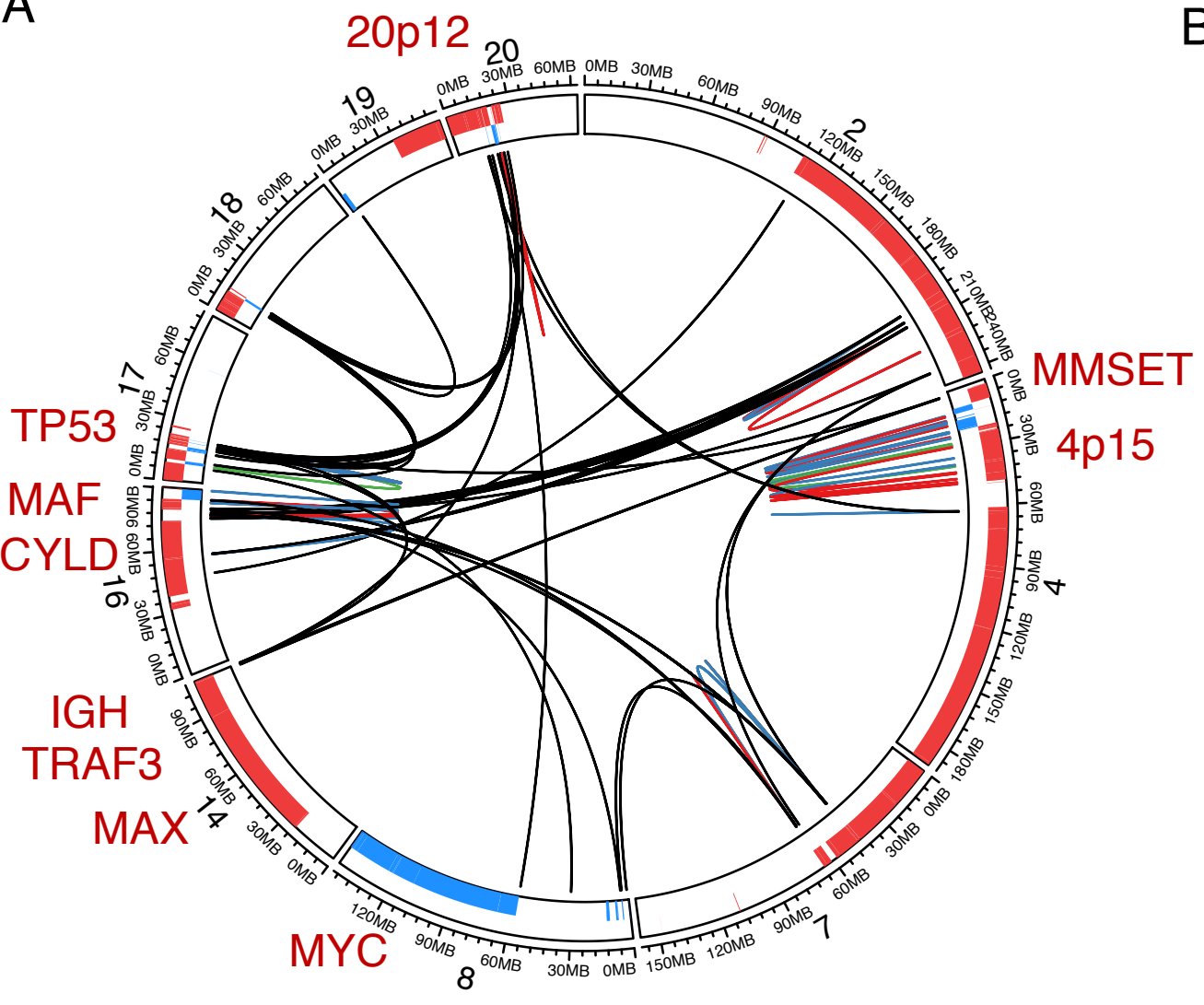

C

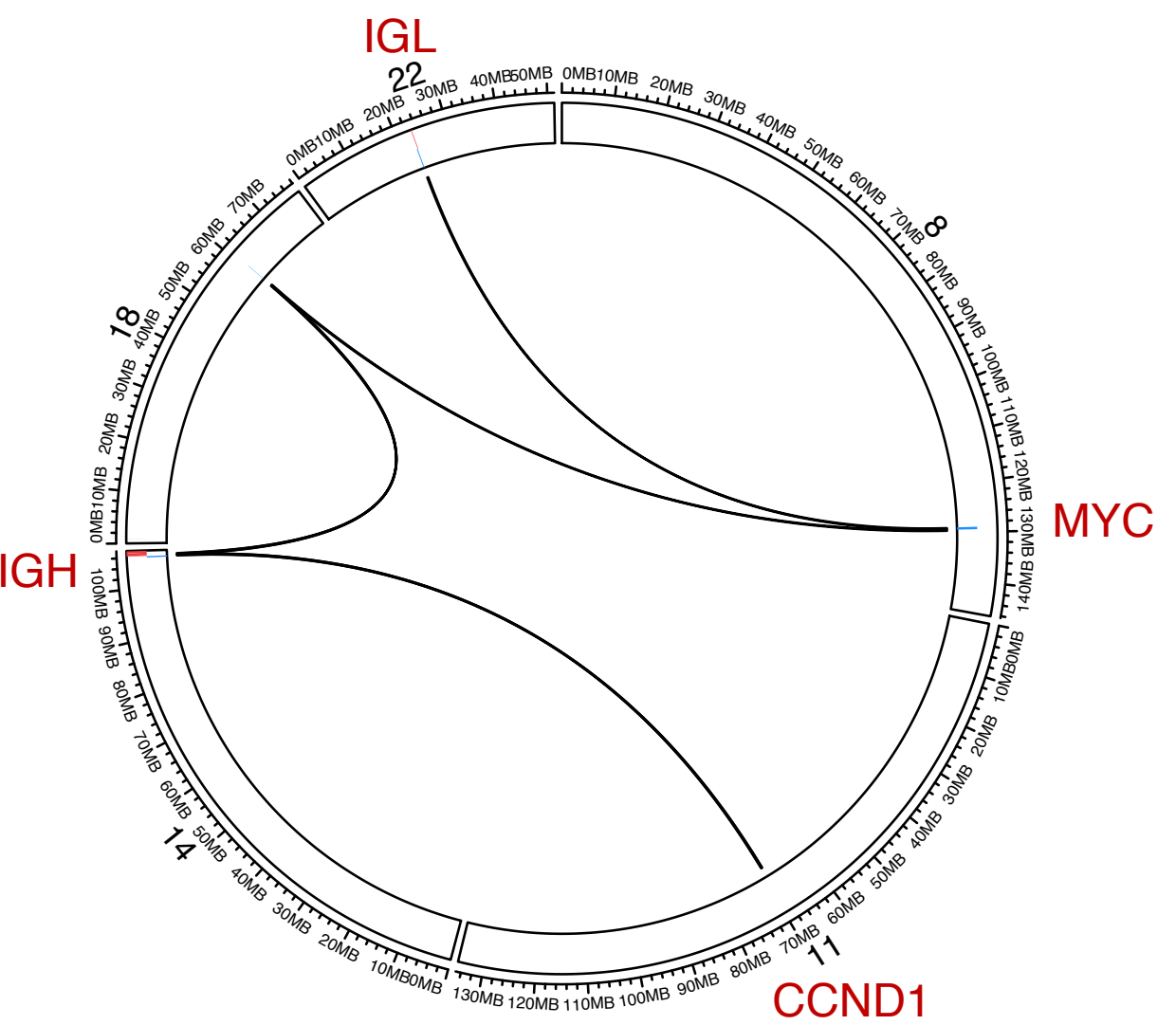

SV classes $\square$ DEL DUP $\square$ INV $\square$ TRA

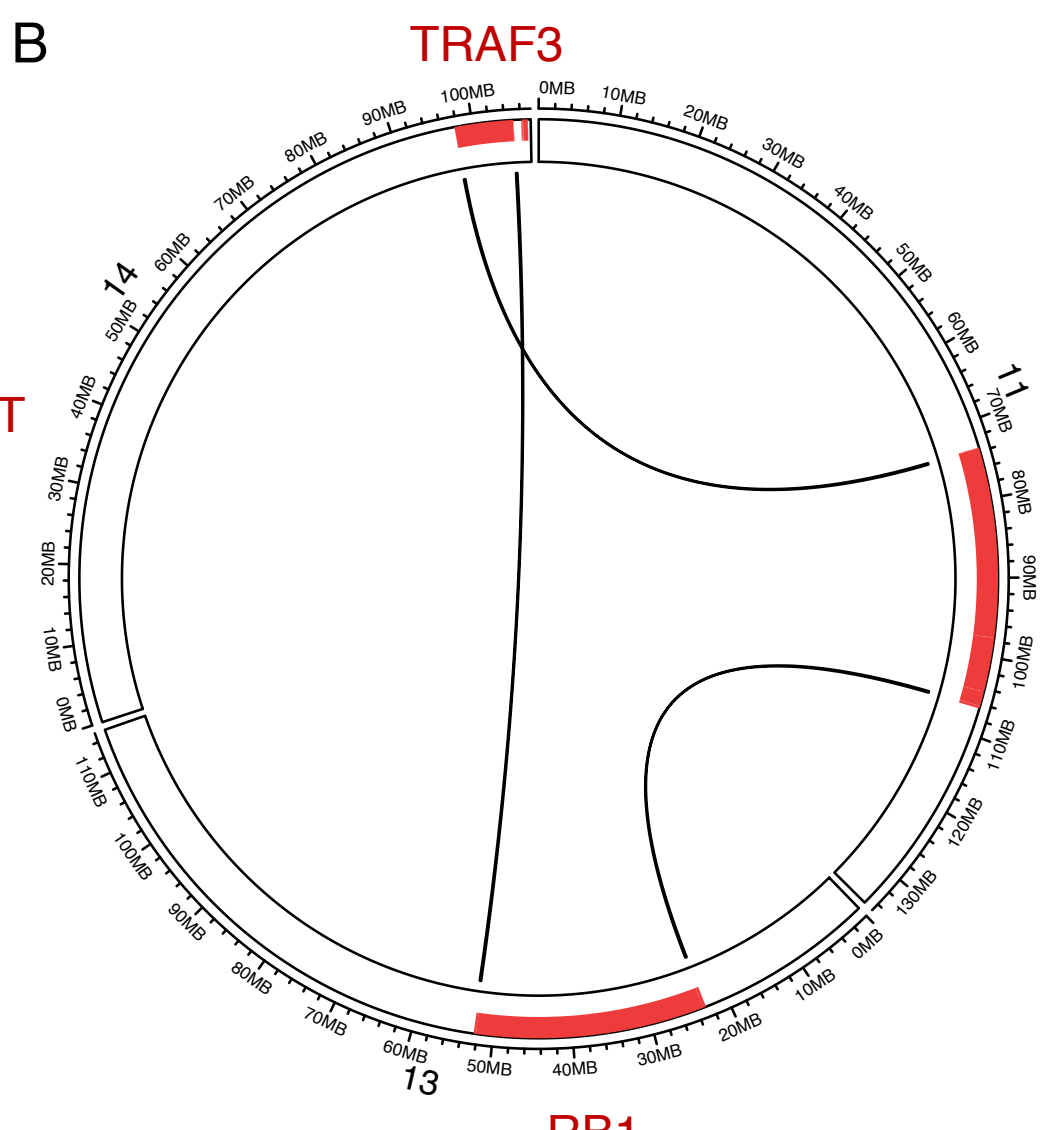

BIRC2 BIRC3

D

$\mathrm{RB} 1$

Chromosome 6

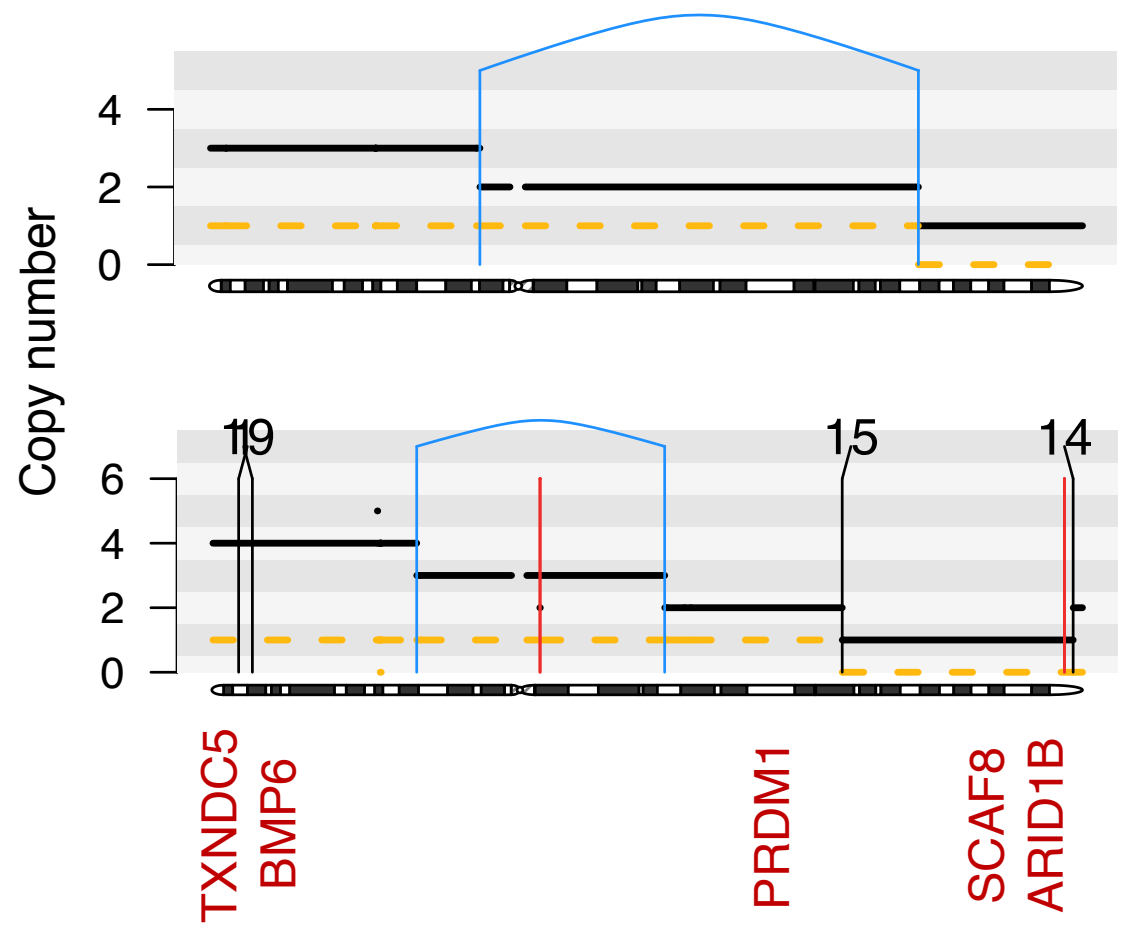


巨ิ $200-$

Gain/Up

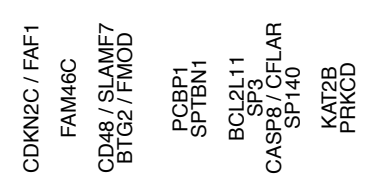

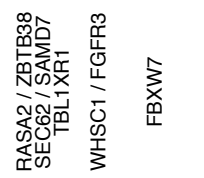

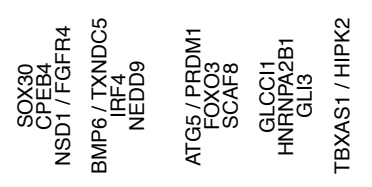
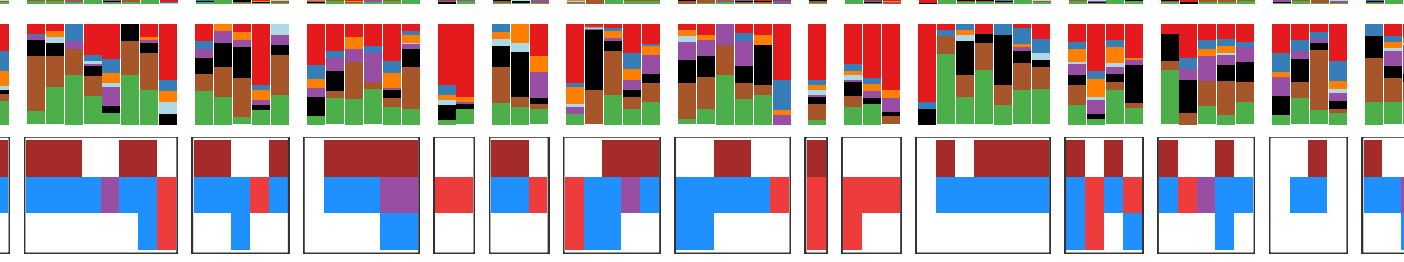

크르-

Loss/Down
Both

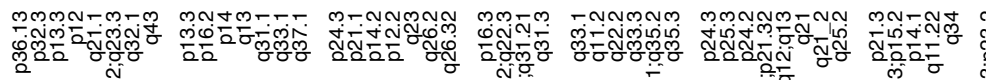
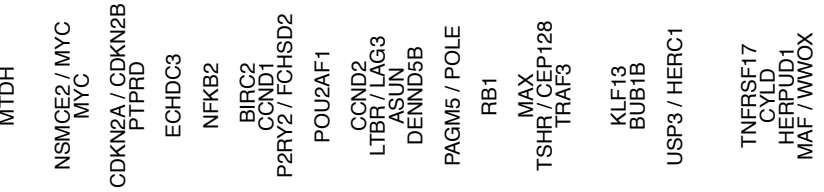

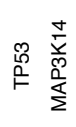

茎

옴름ำ

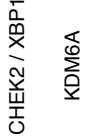

B

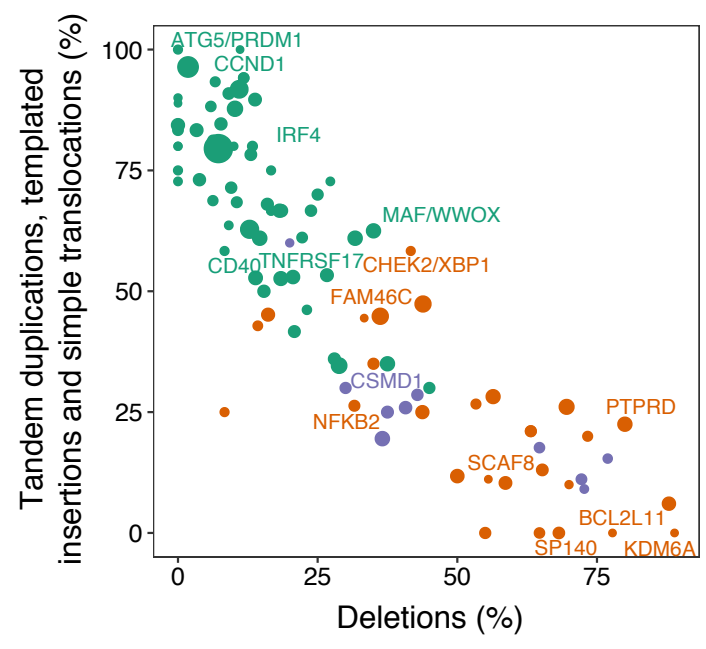

Hotspot class

Gain of function

Loss of function

Unknown

Events ( $\mathrm{n})$

- 10

- 50

100

250

\section{तั}

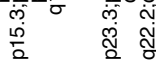

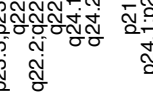

C

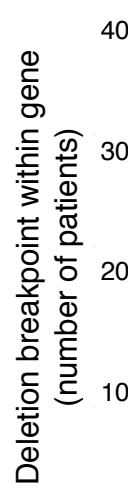

Gene annotations

Gene in validated fragile site

- Tumor suppressor gene

Tumor suppressor \& fragile Neither

Size $(\mathrm{Mb})$

- 0.1

- 1.0

2.0 
Figure 6

A

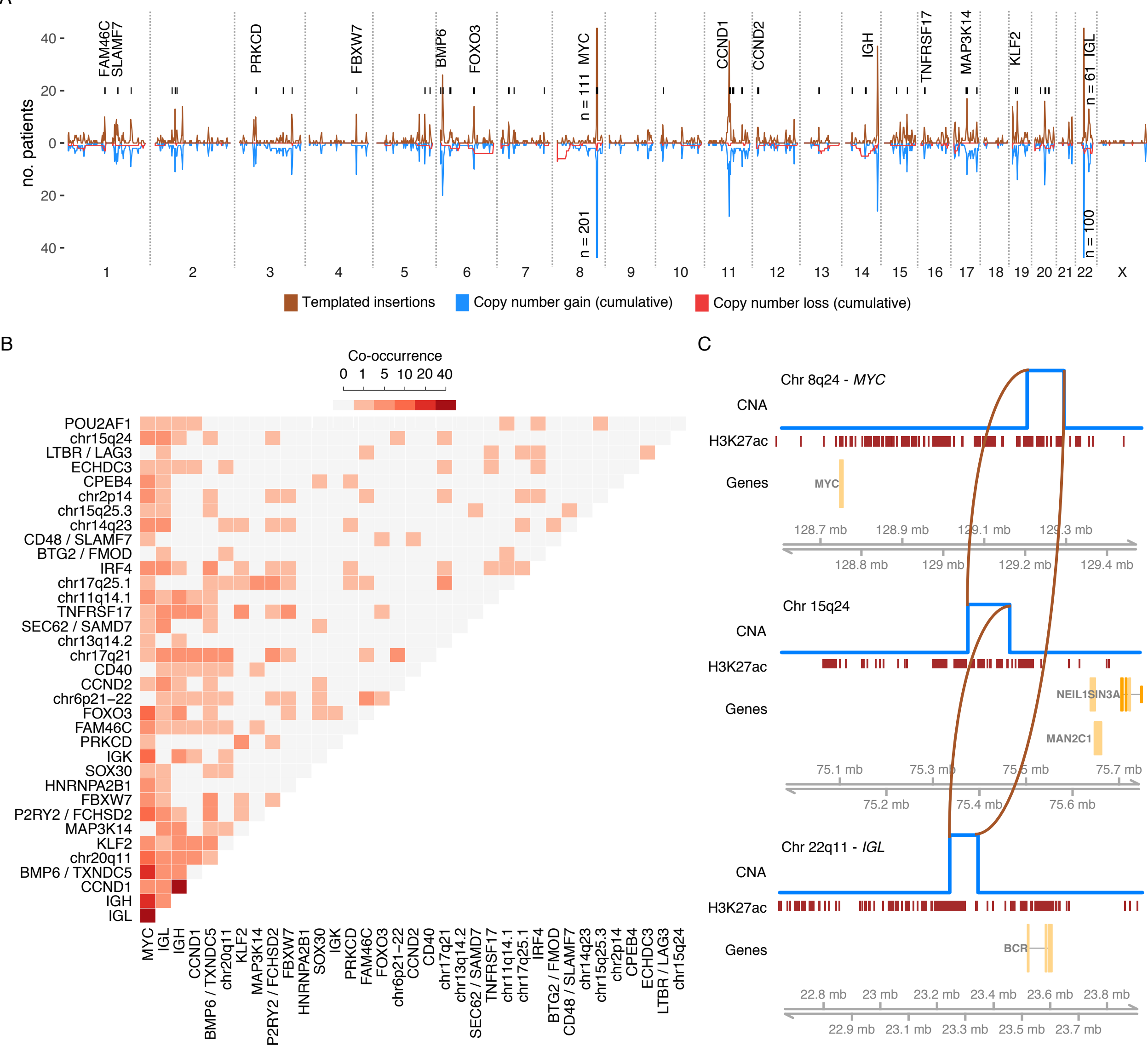




\section{Figure 8}

A

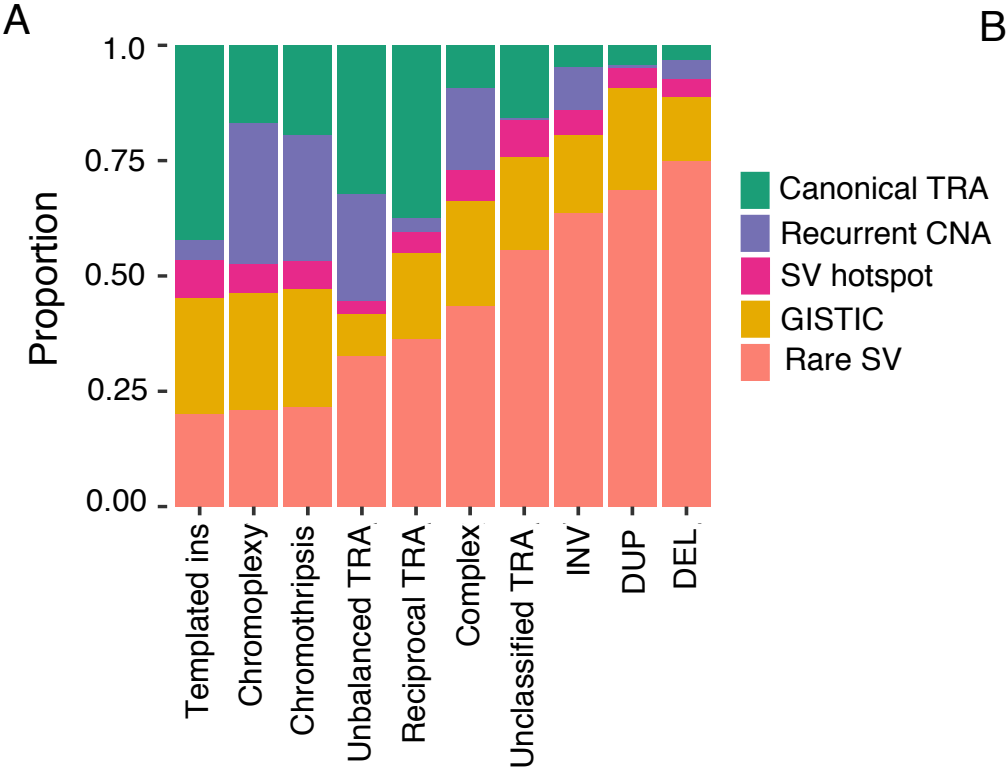

Recurrent SVs

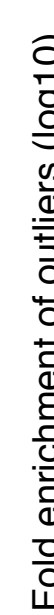
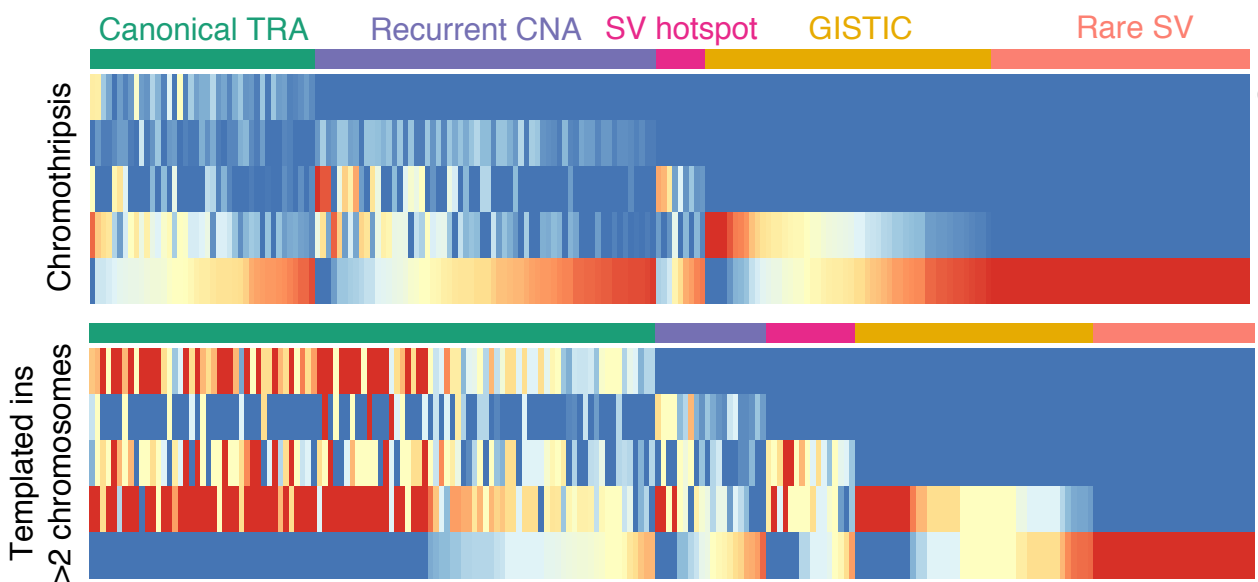

SV breakpoints (prop)

$\begin{array}{llllll}0 & 0.2 & 0.4 & 0.6 & 0.8 & 1\end{array}$
Canonical TRA Recurrent CNA SV hotspot GISTIC Rare SV

Canonical TRA Recurrent CNA SV hotspot GISTIC Rare SV
D

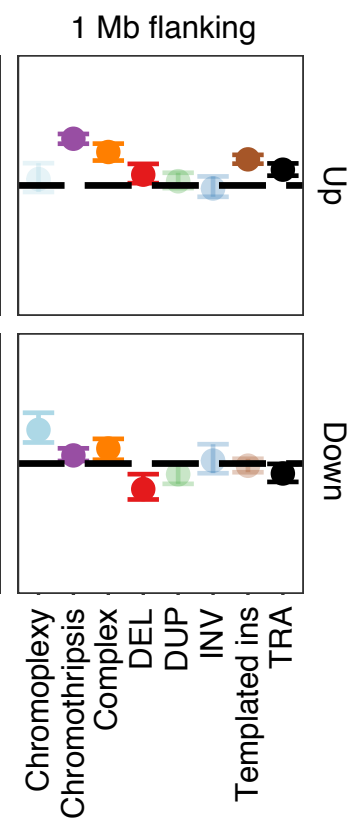

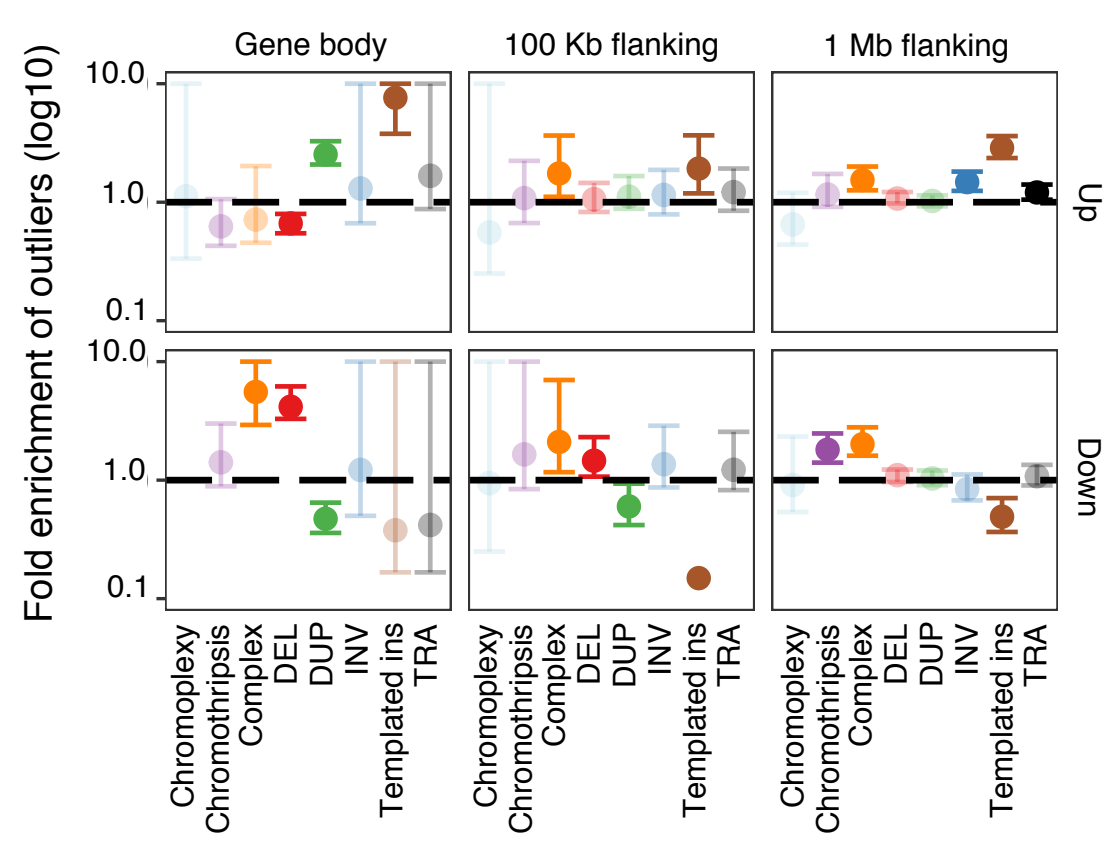

